\title{
Diversidad de la familia Carabidae (Coleoptera) en Chile
}

\author{
Diversity of the family Carabidae (Coleoptera) in Chile
}

\author{
SERGIO ROIG-JUÑENT \& MARTHA C. DOMÍNGUEZ
}

Instituto Argentino de Investigaciones de las Zonas Áridas (IADIZA), Avenida Dr. Adrián Ruiz Leal s/n, Casilla de Correos 507, 5500 Mendoza, Argentina, e-mail: saroig@lab.cricyt.edu.ar

\begin{abstract}
RESUMEN
Carabidae constituye dentro de los coléopteros chilenos, la cuarta familia en importancia por su cantidad de especies. El presente trabajo incluye una breve compilación acerca de la historia de la familia y de las primeras expediciones realizadas en Chile. También se realizan comparaciones de la diversidad de carábidos chilenos con respecto a otros países y el Neotrópico. Para Chile, se conocen 21 tribus, con 95 géneros y 365 especies, que representan el 38,8, 28,8 y 7,9\% de la fauna del Neotrópico, respectivamente. Chile posee un bajo número de tribus comparado con otros países, sin embargo, constituye un área importante por la presencia de seis tribus relictuales, principalmente pangeicas o gondwánicas. Chile posee 18 géneros endémicos (18,5\% de su fauna de Carabidae), 28 cuya distribución está restringida a Chile y Argentina y seis restringidos a Chile, Argentina y Uruguay. La cantidad de especies presentes en Chile es inferior a la que poseen otros países de América del Sur, pero la cantidad de especies endémicas es muy alta (204) y representa el 55,8\% de su fauna de carábidos. El alto grado de endemismo que posee Chile con respecto a otros países de América del Sur puede deberse a su condición de aislamiento, siendo las barreras más importantes la región desértica del norte y la cordillera de Los Andes. Este hecho también se vislumbra por la ausencia de importantes tribus neotropicales como Galeritini, Scaritini y Brachinini. También se incluyen en este trabajo claves para la identificación de todas las tribus y géneros presentes en Chile, como así también una breve descripción acerca de la diversidad y ambientes en los que se encuentra cada género.
\end{abstract}

Palabras clave: Carabidae, Chile, diversidad, taxa endémicos.

\begin{abstract}
The family Carabidae is the fourth largest Coleoptera family in Chile. The present work includes a brief compilation on the taxonomic history of the family and the first expeditions to Chile. In addition, knowledge of carabid diversity in Chile is compared with that of the Neotropics and other South American countries. There are 21 tribes of Carabidae represented in Chile (38.8\% of the total found in Neotropics), with 95 genera ( $28.2 \%$ of the Neotropical fauna), and 365 species ( $7.9 \%$ of the total from the Neotropics). Chile has a low number of tribes compared with other countries, but it is an important area because six relictual tribes occur within it, being mostly gondwanan or pangeic. At the generic level, 18 genera are endemic ( $18.5 \%$ of Chilean genera), 28 genera are restricted to Chile and Argentina, and six to Chile, Argentina, and Uruguay. The number of carabid species in Chile is low compared with other South American countries, but the number of endemic species is high, 204, which is $55.8 \%$ of the total carabid fauna of the country. This high endemicity in Chile might be due to Chile's isolated situation in South America. The Andean mountains and the Northern Desert region, separate Chile from most of the neotropical fauna, as is shown by the absence of important tribes such as the Galeritini, Scaritini, and Brachinini. Keys for all genera present in Chile are provided, with a brief description of habitat and species richness of each.
\end{abstract}

Key words: Carabidae, Chile, diversity, endemic taxa.

\section{INTRODUCCIÓN}

La familia Carabidae (Insecta: Coleoptera) constituye junto con las familias Haliplidae, Hygrobidae, Amphizodidae, Gyrinidae y Dytiscidae el suborden Adephaga, caracterizado por una conformación particular del abdomen (Jeannel \& Paulian 1944).

Los carábidos son quizás uno de los grupos más estudiados, existiendo gran cantidad de investi- gadores que se ocupan de ellos. Entre las razones que motivan este interés, están sus patrones de distribución, la cantidad de taxones relictuales, los numerosos restos fósiles, los compuestos químicos que secretan, la importancia económica y la facilidad para realizar estudios de poblaciones (Ball 1979b).

Se conocen aproximádamente 40.000 especies de carábidos en todo el mundo (Niemelä 1996), de las cuales hay alrededor 4.600 especies en 
América del Sur, distribuidas en siete subfamilias, 51 tribus y 335 géneros (Roig-Juñent 1998a). La familia Carabidae es la cuarta familia más importante en número de especies de Coleoptera en Chile, después de Tenebrionidae (465), Curculionidae (431) y Staphylinidae (424) (Elgueta \& Arriagada 1989). Para Chile, desde la gran obra de Solier (1849) en que se citaban 112 especies en 37 géneros, se llegaron a citar 342 especies y 81 géneros (Elgueta \& Arriagada 1989). Este aumento en la cantidad de taxones no es sólo debido a la descripción de nuevas especies sino también a cambios de jerarquías, sinonimización de nombres y nuevas citas.

Este trabajo pretende brindar una visión actualizada de la riqueza de grupos de Carabidae de Chile, comparándola con la región Neotropical y otros países de América del Sur, como así también analizar la importancia de esta fauna en el contexto del Neotrópico y calcular la riqueza de Carabidae para diversas regiones de Chile.

\section{MATERIALES Y MÉTODOS}

La clasificación utilizada como base para este trabajo es la de Erwin \& Sims (1984), tomando en consideración los cambios propuestos por diversos autores (Roig-Juñent 1998a). Con relación a anteriores catálogos, existe una mayor cantidad de tribus presentes en Chile. Sin embargo, estas tribus nuevas, como Ceroglossini, Platynini y Systolosomini, han sido erigidas para géneros ya existentes y ampliamente conocidos en Chile. Otras tribus se consideran actualmente a nivel de subtribus, tal como Eucheliina (subtribu de Lebiini). Finalmente, Paussinae, Cicindelinae y Trachypachinae, tratadas como familias por Elgueta \& Arriagada (1989) son consideradas en este trabajo como subfamilias o tribus de Carabidae (Anexo 1).

La sección Diversidad Taxonómica se dividió en tres grupos de taxones para facilitar la comparación de la información: supragenéricos, genéricos y específicos.

La información sobre taxones supragenéricos se presenta por tribus, ya que ésta es la forma más corriente de tratar los datos en Carabidae. La disposición de las subfamilias en las tablas y Apéndices sigue un orden filogenético. Dentro de cada subfamilia, las tribus están ordenadas alfabéticamente, de la misma manera que los géneros lo están en las tribus.

Para calcular la cantidad de géneros y especies, se tomaron como base los catálogos de Philippi (1887), Germain (1911) y Blackwelder (1944) y el trabajo de Reichardt (1977), más las revisiones disponibles posteriores al último trabajo. Para los géneros cosmopolitas o con distribución en otros continentes, sólo se consideraron las especies neotropicales, tomando en cuenta aquéllas con distribución al sur de México.

Para las especies del género Ceroglossus Solier, 1849, se consideraron sólo las siete propuestas como válidas por Balazuc (1957). Esto es debido a que este género ha sido uno de los más problemáticos por la cantidad de especies que fueron citadas (ha habido hasta 120 nombres válidos). Se ha tomado esta decisión ya que en algunos casos, por ejemplo en el catálogo de Germain (1911), se citaban 32 especies y 31 variedades, lo cual distorsionaba demasiado la cantidad de especies de Carabidae descritas para Chile.

Muchos de los datos aportados provienen de las colecciones de entomología del Museo de Historia Natural de Santiago (Chile), del Museo de La Plata (Argentina), del Instituto Argentino de Investigaciones de las Zonas Áridas (Argentina) y del Natural History Museum de Londres (Inglaterra). Algunas tribus han sido sido exhaustivamente estudiadas (Cnemalobini y Broscini) y para ello se ha revisado una mayor cantidad de colecciones.

\section{Caracterización de los carábidos}

La familia Carabidae es una de las familias con mayor cantidad de especies en el mundo, con aproximadamente unas 40.000. Dentro del orden Coleoptera, se agrupa con otras familias que constituyen los Hydradephaga (familias Haliplidae, Hygrobidae, Amphizodidae, Gyrinidae y Dytiscidae) en el suborden Adephaga.

Dichas familias son consideradas como un grupo basal dentro de los Coleoptera, constituyendo los Adephaga acuáticos (Hydradephaga) el grupo hermano de Carabidae. Dentro de Carabidae, la subfamilia Trachypachinae (considerada a veces a nivel de familia) se considera como uno de los integrantes del grupo hermano de los restantes carábidos (Roig-Juñent 1998b).

Los fósiles más antiguos de Carabidae datan del Pérmico (Hennig 1981) y se trataría de insectos que habitaban lugares cercanos al agua (Evans 1980), condición compartida por las familias más cercanamente emparentadas y por algunas de las tribus más plesiomorfas de Carabidae como Systolosomini, Trachypachini y Omophronini. Ese linaje pérmico de Carabidae habría ocupado ambientes tropicales húmedos (Erwin 1979), experimentando tempranas radiaciones a otros hábitats, ya que en el Triásico se conocen muchas especies de carábidos con el trocánter alargado, indicando 
que eran especies corredoras de ambientes terrestres (Evans 1980). La amplia distribución de los carábidos y las distintas líneas filéticas existentes, pangeicas, gondwánicas y laurásicas (Jeannel 1942a), también prueban la antigüedad de esas radiaciones evolutivas.

Los carábidos poseen un tamaño corporal desde 1 a $70 \mathrm{~mm}$. La forma de su cuerpo puede ser plana
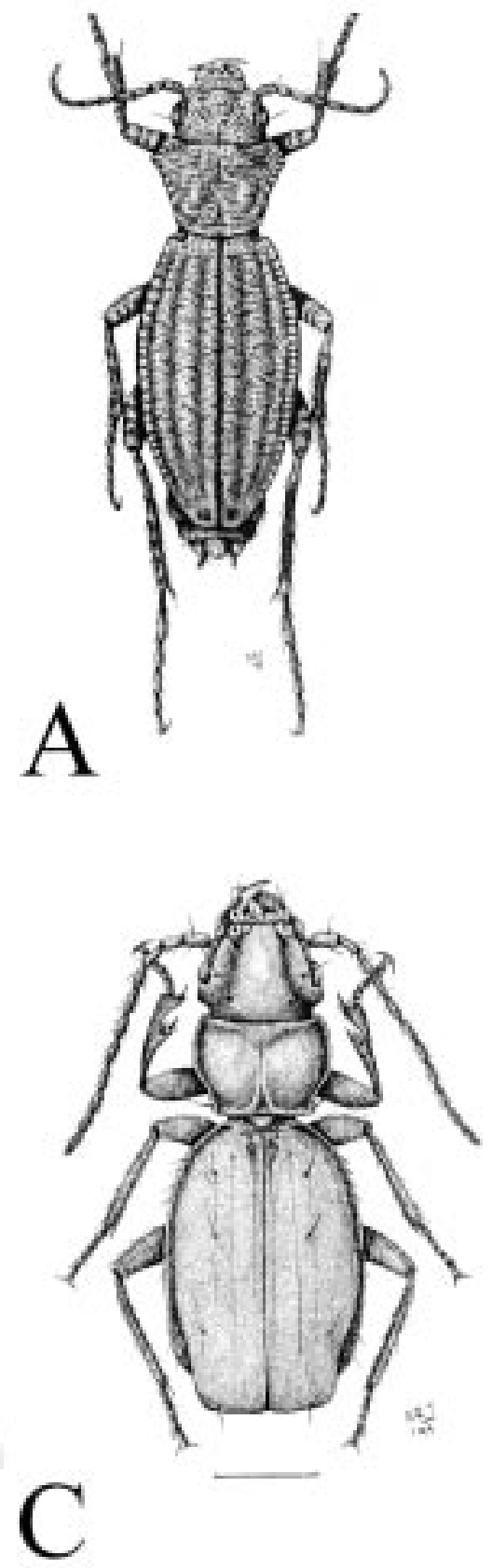

o muy convexa, existiendo gran variedad de formas (Fig. 1A, 1B, 1C y 1D); asimismo, la coloración varía desde totalmente negra a vivos colores metálicos, pero la mayoría es negra o parda oscura.

Sus especies son de ambientes terrestres, aunque existen algunas que habitan ambientes costeros marinos. La gran mayoría de los carábidos son predadores, existiendo algunas especies
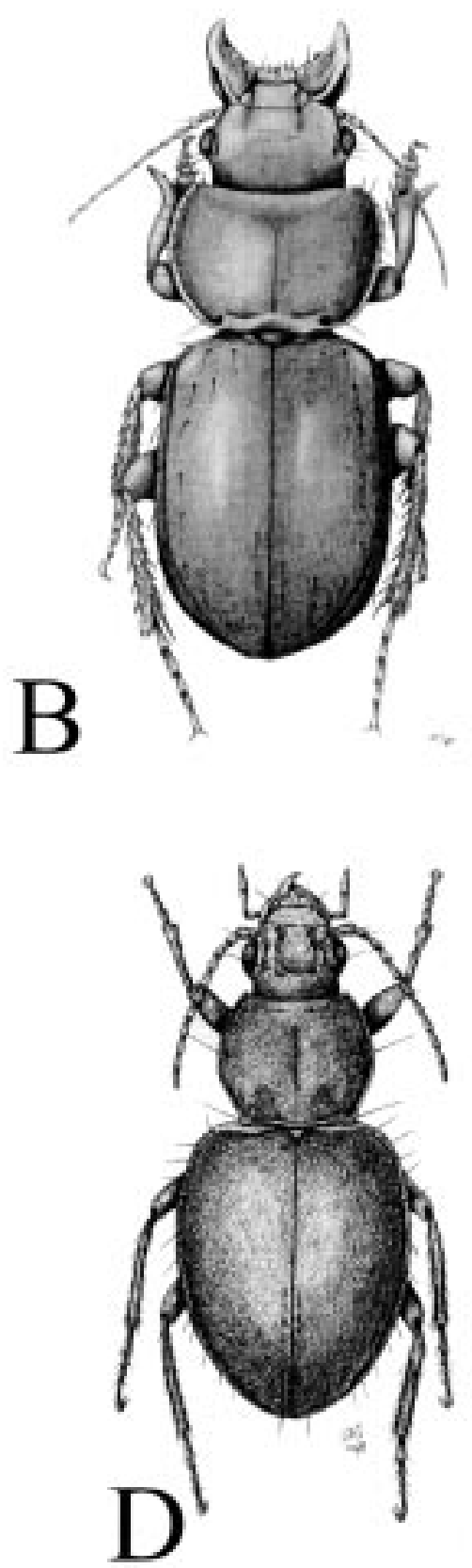

Fig. 1: Vista dorsal de (A) Cnemalobus montanus, (B) Pycnochila fallaciosa, (C) Tropopterus giraudi y (D) Kenodactylus audouini.

Dorsal view of (A) Cnemalobus montanus, (B) Pycnochila fallaciosa, (C) Tropopterus giraudi and (D) Kenodactylus audouini. 
fitófagas (Zabrini y algunos Harpalini). Los adultos poseen una amplia gama de sistemas químicos de defensa (Moore 1979).

Las larvas son terrestres a diferencia de los demás adéfagos, cuyas larvas son acuáticas (Thompson 1979). El estado larval posee tres estadios y su régimen alimenticio es amplio; la mayoría son predadores, pudiendo algunos alimentarse de frutas o semillas, ser comensales de hormigas o ectoparásitos de pupas de coleópteros.

Los carábidos son insectos muy abundantes en ecosistemas húmedos, siendo su diversidad menor en ambientes templados áridos (Erwin 1985). Pueden pertenecer principalmente a tres grupos ecológicos: higrófilos, que viven en los bordes de arroyos o estanques; arborícolas, que viven en troncos u hojas; y geófilos, que viven en el suelo sin estar asociados al agua. Estos últimos pueden constituir elementos de la macrofauna de suelo, endógeos o cavernícolas. Por su régimen alimenticio, ciclos de vida y preferencias ambientales, son objeto de numerosos estudios (Thiele 1977). Dentro de los agroecosistemas, constituyen un elemento importante al ser controladores de numerosas plagas (Allen 1979).

Esta familia de Coleoptera ha sido muy utilizada para realizar inferencias biogeográficas (Jeannel 1942a, Darlington 1965, Kavanaugh 1979a, Noonan 1979), como así también para realizar estudios acerca de patrones y procesos de especiación (Kavanaugh 1979b).

\section{Historia taxonómica}

La familia Carabidae fue creada por Latreille en 1802 como la tercera familia del grupo de coleópteros pentámeros, constituida por 29 géneros agrupados en siete tribus. Posteriormente, comenzó un período en el que los viajes alrededor del mundo aportaron nuevos materiales, aumentando notablemente el número de especies y géneros conocidos del grupo, por lo que las siete tribus establecidas por Latreille no fueron suficientes y comenzaron a crearse nuevas tribus (Dejean 1825-1831, Lacordaire 1854). Sin embargo, este aumento del número de taxones fue sólo cuantitativo y es a partir de los trabajos de Chaudoir $(1854,1861)$ que se trató de establecer una clasificación de la familia. El siguiente sistema de clasificación importante de Carabidae lo realizó Sloane (1923) y, posteriormente, Jeannel (1941, 1942b) reordenó los grupos de Carabidae de acuerdo a lo que él llamó linajes, introduciendo una visión evolucionista en la clasificación. Actualmente, existen 85 tribus de Carabidae (Erwin \& Sims 1984) y hay un gran consenso entre los especialistas acerca de las relaciones entre los distintos grupos que conforman la familia, facilitando la elaboración de una clasificación que refleja la filogenia de la familia (Ball 1979a).

Primeros viajes de recolección y descripción de carábidos de Chile

Hasta el año 1825 , se había publicado muy poco sobre Carabidae neotropicales, sólo unas 59 especies descritas de 25 géneros (Tabla 1). Los primeros que describieron especies de Chile, hasta 1825 , fueron Linneo y Fabricius, conociéndose sólo cuatro especies: Megacephala carolina Linneo, 1766 (América meridional), Abropus carnifex Fabricius, 1775 (América meridional), Ceroglossus suturalis (Fabricius, 1775) (Tierra del Fuego) y Trirammatus striatula (Fabricius, 1775) (América meridional). No es de extrañar que estas especies llamaran la atención de los coleccionistas, pues algunas son de gran tamaño o de colores muy brillantes. Ningún carábido de pequeño tamaño fue descrito para la región austral de América del Sur en este período (Roig-Juñent 1998a).

Como sucedió para el resto de la región Neotropical (Ball 1996), la obra de Dejean (18251831) aumentó notablemente la cantidad de especies conocidas para Chile (entre 1828-1831 describió 23 especies). Otros autores pioneros en la descripción de especies chilenas fueron Eschelhotz, Laporte y Hope. Sin embargo, en este período tuvieron mayor trascendencia las publicaciones como resultados de viajes o expediciones científicas. Así, Guérin-Ménéville describió varias especies colectadas por la expedición de "La Favorite" y "La Coquille", mientras que Curtis y Waterhouse describieron gran cantidad de especies nuevas de la Patagonia a partir de lo colectado por Darwin en su viaje del "Beagle" y por King en el "Endeavour"; Brullé describió material colectado por D' Orbigny en el "Voyage dans 1' Amérique Meridionale". Entre 1838 y 1849 se describieron 63 especies de Carabidae para Chile.

A pesar del incremento del conocimiento por viajes de europeos, el mayor aporte a la fauna de Carabidae chilena por esa época fue la obra de Solier (1849) como parte de la Historia Física y Política de Chile de Claudio Gay, elevando a 37 la cantidad de géneros para Chile e incluyendo 112 especies (Tabla 1). Solier describió en esta obra 16 géneros como nuevos (de los cuales 13 son actualmente válidos) y 82 nuevas especies (de las cuales se mantienen 63). Con posterioridad a esta obra, fueron muy escasos los aportes realizados por investigadores europeos. La siguiente obra de importancia la realizó Reed (1874), quién publicó 
un trabajo exclusivo de Carabidae chilenos y sintetizó el conocimiento de esta familia para ese momento, describiendo algunos géneros y especies nuevas; en total citó 50 géneros con 165 especies.

Posteriores a la obra de Reed, las únicas expediciones científicas europeas fueron las de "La Romache" y la "Mission Scientifique du Cap Horn", siendo Fairmaire entre 1883 y 1888 quién describió diversas especies, la mayoría de la Patagonia. Chile no escapa al resto del Neotrópico $\mathrm{y}$, como lo hace notar Ball (1996), entre 1885 y 1924 fue el período en que menos géneros de Carabidae fueron descritos para el Neotrópico (tan sólo 16, mientras que en 1825-1884 fueron 155 y entre 1925 y 1955 fueron 112). El menor número de géneros descritos en el período 1885 1924 se debería, según Ball (1996), al interés de los europeos por África y el lejano Oriente y a las dificultades creadas por el comienzo de guerras entre países europeos, como la Franco-Prusiana en 1879 y la primera guerra mundial en 1914.

\section{TABLA 1}

Tribus de Carabidae con la cantidad de géneros y especies (entre paréntesis) citados para Chile y el Neotrópico. Los paréntesis cuadrados indican el número de nomen nuda citados

Carabid tribes with the number of genera and species (in parenthesis) cited for Chile and the Neotropics. Squared parentheses indicate the number of nomen nuda cited

\begin{tabular}{|c|c|c|c|c|c|c|c|c|c|c|}
\hline \multirow[t]{2}{*}{ Tribu } & \multicolumn{2}{|c|}{ Antes de 1825} & \multirow{2}{*}{$\begin{array}{c}\text { Solier } \\
1849 \\
\text { Chile }\end{array}$} & \multirow{2}{*}{$\begin{array}{l}\text { Reed } \\
1874 \\
\text { Chile }\end{array}$} & \multirow{2}{*}{$\begin{array}{c}\text { Philippi } \\
1887 \\
\text { Chile }\end{array}$} & \multirow{2}{*}{$\begin{array}{c}\text { Germain } \\
1911 \\
\text { Chile }\end{array}$} & \multicolumn{2}{|c|}{ Blackwelder 1944} & \multicolumn{2}{|c|}{ Posterior a 1944} \\
\hline & Neot & Chile & & & & & Neot & Chile & Neot & Chile \\
\hline \multicolumn{11}{|l|}{ Trachypachinae } \\
\hline Systolosomini & & & $1(1)$ & $1(1)$ & $1(1)$ & $1(2)[1]$ & $1(1)$ & $1(1)$ & $1(2)$ & $1(2)$ \\
\hline \multicolumn{11}{|l|}{ Carabinae } \\
\hline Carabini & $1(2)$ & & $1(1)$ & $1(1)$ & $1(1)$ & $1(1)$ & $2(16)$ & $1(1)$ & $1(61)$ & $1(4)$ \\
\hline Ceroglossini & $1(1)$ & $1(1)$ & $1(3)$ & $1(6)$ & $1(6)$ & $1(6)$ & $1(6)$ & $1(6)$ & $1(8)$ & $1(8)$ \\
\hline Cicindelini & $2(4)$ & $1(1)$ & $1(2)$ & 1(3) & 1(3) & $1(3)$ & $7(68)$ & $1(2)$ & $8(170)$ & $1(5)$ \\
\hline Megacephalini & $1(2)$ & & $1(1)$ & $1(1)$ & $2(2)$ & $2(3)$ & $8(74)$ & $2(2)$ & $7(73)$ & $2(3)$ \\
\hline Migadopini & & & $1(1)$ & $4(7)$ & $6(10)$ & $3(5)$ & $7(11)$ & $6(8)$ & $7(14)$ & $6(12)$ \\
\hline \multicolumn{11}{|l|}{ Paussinae } \\
\hline Ozaenini & $1(1)$ & & $1(4)$ & $1(3)$ & $1(4)$ & $1(4)[1]$ & $6(78)$ & $2(3)$ & $7(87)$ & $2(4)$ \\
\hline \multicolumn{11}{|l|}{ Psydrinae } \\
\hline Bembidiini & & & $1(18)$ & $3(21)$ & $3(21)$ & $1(34)[2]$ & $8(226)$ & $2(42)$ & $32(250)$ & $17(71)$ \\
\hline Psydrini & & & $1(2)$ & $1(4)$ & $1(4)$ & $1(4)[1]$ & $2(4)$ & $2(4)$ & $3(5)$ & $2(4)$ \\
\hline Trechini & & & $3(5)$ & $3(22)$ & $4(30)$ & $3[1] 17[7]$ & $10(49)$ & $7(22)$ & $14(100)$ & $11(56)$ \\
\hline Zolini & & & $1(1)$ & $1(1)$ & $1(2)$ & $1(1)$ & $1(2)$ & $1(2)$ & $2(4)$ & $1(3)$ \\
\hline \multicolumn{11}{|l|}{ Broscinae } \\
\hline Broscini & $1(1)$ & & $2(4)$ & $2(7)$ & $2(9)$ & $2(10)[2]$ & $3(14)$ & $3(8)$ & $5(28)$ & $6(12)$ \\
\hline \multicolumn{11}{|l|}{ Harpalinae } \\
\hline Chlaenini & $1(1)$ & & & $1(2)$ & & & $1(31)$ & $1(1)$ & $1(20)$ & $1(1)$ \\
\hline Ctenodactilini & & & $1(1)$ & $1(1)$ & $1(1)$ & $1(1)$ & $14(64)$ & $1(1)$ & $15(112)$ & $1(1)$ \\
\hline Cnemalobini & & & $1(4)$ & $1(4)$ & $1(11)$ & $1(6)$ & $2(17)$ & $2(15)$ & $1(23)$ & $1(17)$ \\
\hline Harpalini & $2(4)$ & & $5(18)$ & $6(19)$ & $9(26)$ & $6(21)[4]$ & $26(320)$ & $8(20)$ & $25(354)$ & $11(32)$ \\
\hline Lebiini & $5(27)$ & & $7(15)$ & $10(21)$ & $10(24)$ & $6(26)[5]$ & $58(916)$ & $10(30)$ & $65(133)$ & $17(51)$ \\
\hline Licinini & & & $1(1)$ & $1(1)$ & $1(1)$ & $1(1)$ & $3(5)$ & $1(1)$ & $4(10)$ & $1(2)$ \\
\hline Masoreini & & & $1(1)$ & $1(2)$ & $1(11)$ & $1(1)$ & $5(44)$ & $2(2)$ & $3(47)$ & $1(2)$ \\
\hline Platynini & & & $2(8)$ & $2(9)$ & $3(11)$ & $2(8)[1]$ & $8(300)$ & $3(12)$ & $13(305)$ & $3(11)$ \\
\hline Pterostichini & $3(3)$ & $2(2)$ & $4(22)$ & $8(31)$ & $3(59)$ & $3(40)[13]$ & $35(333)$ & $5(52)$ & $33(454)$ & $8(64)$ \\
\hline $\begin{array}{l}\text { Otras tribus no } \\
\text { presentes en Chile }\end{array}$ & $7(13)$ & & & & & & $100(960)$ & & $88(1.119)$ & \\
\hline Total & $25(59)$ & $4(4)$ & $37(112)$ & $50(165)$ & $54(239)$ & $39[1] 194[37]$ & $308(3.539$ & $64(237)$ & $336(4.585$ & 5) $95(365)$ \\
\hline
\end{tabular}


Sin embargo, para Chile comienzan a tener importancia investigadores que trabajaban en el país. Después de Carlos Reed, hubo dos importantes investigadores, R. A. Philippi y P. Germain. Philippi realizó una expedición al Sur (Philippi \& Juliet 1871) y describió un número importante de especies (Philippi 1887) y también amplió el área de distribución de numerosas especies, antes no conocidas para Chile. Germain describió una gran cantidad de especies, como así también realizó numerosas revisiones sistemáticas (Germain 1855a, 1855b, 1893, 1901, 1905, 1911). Durante este período, se aumentó considerablemente el conocimiento de los carábidos chilenos, sobre todo de los grupos tratados por Germain. Desafortunadamente, el catálogo que publicó Germain (1911) solo trató los materiales depositados en el Museo de Historia Natural de Santiago e incluso numerosas especies (37) citadas en él constituyen nomen nuda. Después de este investigador y hasta la actualidad, muy pocos trabajos realizados por chilenos versaron sobre carábidos, excepto los de Ruiz-Pereira (1937) y Peña \& Barría-Pereira (1973). Entre investigadores de otros países de América del Sur que estudiaron Carabidae cabe mencionar a Reichardt de Brasil y muy recientemente el primer autor de este artículo.

El interés por los Carabidae de Chile no se reinició inmediatamente después de la primera guerra mundial, como cita Ball (1966) para el Neotrópico, sino recién en 1938 y la década de 1960 , por René Jeannel, quién fue el primer investigador en tratar grupos presentes en Chile. En este mismo período, se reinicia el interés de los extranjeros por América del Sur, debido a nuevas excursiones como la de Straneo (1969) o la realizada a las islas Juan Fernández (Straneo \& Jeannel 1955). A partir de la decada de 1970 , comienzan a realizarse numerosas revisiones, tanto por investigadores chilenos (Peña \& Barría-Pereira 1973) como por numerosos investigadores extranjeros como Ball, Darlington, van Emdem, Erwin, Jeannel, Liebke, Mateu, Nègre, Noonan, Reichardt y Straneo. En este período, se publican gran cantidad de artículos referidos a Carabidae neotropicales que incluyen especies presentes en Chile.

Al igual que para el neotrópico una obra de gran importancia es la sinopsis de los géneros neotropicales de Reichardt (1977) compilada por George Ball, quién actualizó la información existente de todos los géneros conocidos para América del Sur, incluyendo claves para su reconocimiento y notas críticas para cada uno de ellos. Sobre esta obra esta basada gran parte de este trabajo.

\section{Catálogos}

Los carábidos chilenos casi siempre se trataron conjuntamente con otros taxones en los catálogos mundiales (Gemminger \& Harold 1868, Csiki 1927-1933), latinoamericanos (Blackwelder 1944), de una porción del continente (Kolbe 1907, Enderlein 1912) y asimismo en el catálogo de Coleópteros Chilenos de Philippi (1887) y de Germain (1911). La única excepción a ello es el trabajo de Reed (1874) de los Geodephaga de Chile.

Reed (1874) citaba para Chile 50 géneros y 171 especies (Tabla 1); posteriormente, Philippi (1887) elevó la cantidad de taxones a 54 géneros y 239 especies. La menor cantidad de géneros y especies citadas 24 años más tarde por Germain (1911) (39 géneros y 188 especies) se debe a que este autor hizo tan sólo un catálogo de los materiales del Museo de Historia Natural de Santiago. Asimismo, hay que hacer notar que si no se consideran los nomen nuda publicados por Germain (37 en total, Tabla 1) sería mucho menor la cantidad de especies. Finalmente el último catálogo (Blackweleder 1944) cita para Chile 64 géneros y 240 especies.

Tribus y géneros citados para Chile considerados como citas erróneas

Hay que hacer especial referencia a dos taxones citados por Phillipi (1887) como presentes en Chile y que sin duda constituyen un error de localidad. Varios de ellos deben deberse a un error de rotulación por parte de Guérin-Ménéville (1838). Philippi cita un Galeritini para Magallanes, Galerita lacordairei Dejean, 1826 (= Galerita magellanica Guérin-Ménéville, 1838, localidad tipo: "Détroit de Magellan"). Esta localidad citada debe constituir un error de rotulación, pues esta especie, muy abundante, se encuentra distribuida al Norte de las provincias argentinas de Mendoza, La Pampa y Buenos Aires, unos $2.000 \mathrm{~km}$ al Norte de la localidad tipo. De igual manera Phillipi (1887) cita para Magallanes a un Scaritini, Scarites melanarius Dejean, 1831 (= Scarites magellanicus GuérinMénéville, 1838, localidad tipo: "Détroit de Magellan"). Esta especie posee una distribución semejante a la anterior, por lo que la localidad de Guérin-Ménéville debe considerarse errónea.

Otra cita que debe considerarse errónea es la de Brachinus intermedius Brullé, 1838 (Brachinini). Blackwelder (1944) cita como dudosa su presencia en Chile. Esta especie se encuentra distribuida en Uruguay, Brasil y en Argentina sólo en la 
provincia de Buenos Aires, por lo que la cita de Chile es probable constituya un error.

\section{DIVERSIDAD TAXONÓMICA}

\section{Taxa supragenéricos}

Chile posee representantes de seis de las siete subfamilias de Carabidae y de 21 tribus, lo cual representa un $24,7 \%$ de las 85 tribus citadas por Erwin \& Sims (1984) para todo el mundo y un $38,8 \%$ de las 51 citadas para la región Neotropical. Esta representatividad es baja para América del Sur, puesto que por ejemplo para Argentina hay citadas 37 tribus $(72,5 \%$ del Neotrópico) y para Brasil 33 (64,7 \% del Neotrópico). Es incluso notable la ausencia de grupos neotropicales ampliamente distribuidos en América del Sur, como Galeritini, Pseudomorphini, Colliurini, Brachinini o Scaritini. La ausencia de ésta última es la más llamativa aún, puesto que constituye un grupo con gran cantidad de especies en Argentina (RoigJuñent 1998a).

\section{Tribus y subtribus endémicas o relictuales}

Si bien ninguna tribu es endémica para Chile, existen muchas tribus o subtribus endémicas de América del Sur (Reichardt 1979) que son compartidas sólo entre Chile y Argentina o entre Chile, Argentina y Uruguay:

(1) Systolosomini. Argentina y Chile: dos especies de Systolosoma Solier, 1849 de los bosques de Nothofagus Blume relacionadas con Trachypachini, de distribución paleártica.

(2) Ceroglossini. Argentina y Chile: ocho especies de los bosques de Nothofagus. Las relaciones de esta tribu con otras son inciertas.

(3) Cnemalobini (Fig. 1A). Argentina, Chile y Uruguay: 23 especies. Relacionada con la tribu holártica Zabrini y la tribu tropical Morionini (Roig-Juñent 1993).

Existen otros taxa supragenéricos presentes en Chile que no son endémicos de América del Sur, pero que poseen una distribución relictual:

(1) Broscini: Es una tribu de distribución anfitropical (Roig-Juñent, 2000) con una distribución en casi todas las áreas australes del mundo, excepto en Africa del Sur. En América del Sur, está restringida a Chile, Argentina y Uruguay.

(2) Omina (subtribu de Cicindelini) (Fig. 1B): una especie, Pycnochilla fallaciosa (Chevrolat, 1854) del Sur de Patagonia, relacionada con dos géneros del oeste de los Estados Unidos.
(3) Zolini. Tribu restringida a Australia, Tasmania, Nueva Zelanda y tres especies de Patagonia (Argentina y Chile). Dos géneros para América del Sur (Roig-Juñent \& Cicchino en prensa), uno solo presente en Chile con tres especies. Tribu relacionada con Trechini y Bembidiini.

(4) Migadopini. Distribución semejante a la anterior, en Argentina, Chile y Uruguay: 12 especies. Relacionada con la tribu holártica Elaphrini (Roig-Juñent 1998b).

\section{Cantidad de especies por tribu}

La mayor diversidad de especies en Chile corresponde a cinco tribus (Tabla 1): Bembidiini (71 en Chile y 250 en la región Neotropical); Pterostichini (64 especies en Chile y 454 en la región Neotropical); Trechini (56 en Chile y 100 en la región Neotropical); Lebiini (51 en Chile y 1339 en la región Neotropical) y Harpalini (32 en Chile y 354 en la región Neptropical). Las restantes tribus poseen menos de 18 especies cada una. $\mathrm{Si}$ bien estas tribus tienen una menor cantidad de especies presentes en Chile, para algunas de ellas (como Cnemalobini, Migadopini, Broscini y Zolini) Chile es el país de América del Sur donde están mejor representadas (Tabla 1 y Anexo 1), ya sea por la cantidad de especies o por la representatividad de los géneros de cada tribu.

\section{Taxa genéricos}

Hay 95 géneros citados para Chile (Tabla 1), que representan el 28,2 \% de los 336 géneros neotropicales. Esta cantidad es baja si se considera que para Argentina hay citados 170 géneros $(50,5 \%$ de la región Neotropical) y para Brasil 194 (57,7 \% de la región Neotropical). En el Anaxo 1 se da una clave para la identificación de todos los géneros presentes en Chile, citando además el ambiente que ocupan, la cantidad de especies por género, las presentes en Chile y la cantidad de ellas que son endémicas.

\section{Géneros endémicos de Chile y distribución en países limítrofes}

Existen 18 géneros endémicos de Chile, 13 de ellos son monoespecíficos y cinco tienen dos especies. Esto es un muy alto grado de endemismo ( $18,9 \%$ de su fauna), puesto que para Argentina sólo hay citados nueve géneros endémicos $(5,5 \%$ de su fauna) (Roig-Juñent 1998a). Este grado de endemismo se ve notablemente aumentado al con- 
siderar géneros con distribuciones restringidas. Así, existen géneros patagónicos que se encuentran sólo en Argentina y Chile (28 géneros) o Argentina, Chile y Uruguay (seis) y que constituyen el 35,7 \% de los 95 géneros de Chile. Sumando los géneros exclusivos de Chile más aquellos compartidos con países limítrofes, el 54,7\% de los géneros de Chile sólo se encuentran en su territorio o en áreas limítrofes, la mayoría en los bosques de Nothofagus.

\section{Cantidad de especies por género}

Ocho géneros poseen la mayor cantidad de especies en Chile: Trechisibus Motschulsky, 1862 (37), Metius (21), Cnemalobus Guérin-Méneville, 1838 (17), Notaphus Stephens, 1928 (16), Trirammatus Chaudoir, 1838 (16), Mimodromius Chaudoir, 1873 (15), Parhypates Motschulsky, 1865 (14) y Peryphus Stephens, 1928 (13). Estos géneros presentan una mayor o igual diversidad en Chile que en otros países (en Argentina Metius tiene 24 especies y Trirammatus 15).

De los géneros restantes, 14 poseen entre cinco y 10 especies en Chile. Entre estos géneros se encuentran cinco que poseen todas o casi todas sus especies en Chile, como Trachysarus Reed, 1874, Notaphiellus Jeannel, 1962, Notholopha Jeannel, 1962, Ceroglossus Solier, 1849 y Nothocys Jeannel, 1962 (Anexo 1).

Los restantes 73 géneros poseen menos de 10 especies presentes en Chile. Sin embargo, esta gran cantidad de géneros con pocas especies no indica en absoluto una baja diversidad, puesto que 28 de ellos son monespecíficos, 11 poseen dos especies, ambas presentes en Chile, uno con dos especies una de ellas en Chile y cinco poseen de tres a cuatro especies, todas ellas presentes en Chile.

Es decir que de los 95 géneros presentes en Chile, 66 están bien representados. Los restantes 32 géneros estarían poco representados. Hay que hacer notar que existen cinco de estos géneros que son de origen holártico, con una especie en Chile que ha sido introducida (Erwin 1978) (Anexo $1)$.

\section{Taxa específicos}

Se contabilizaron 365 especies (Tabla 1) que representan el 7,9\% de las 4.585 especies neotropicales. Esta cantidad representa una diversidad baja, puesto que para Argentina hay 683 especies (14,8\% del Neotrópico) y para Brasil 1.506 (33,9\% del Neotrópico).

\section{Especies endémicas}

Existen 204 especies citadas sólo para Chile (Tabla 1). Es muy probable que muchas de ellas se hallen en países limítrofes, pero hasta el momento su distribución conocida se restringe a Chile. Este porcentaje $(55,8 \%)$ es alto puesto que Argentina, que posee casi el doble de especies, tiene sólo 278 endémicas $(40,8 \%)$.

\section{ESTADO ACTUAL DEL CONOCIMIENTO DE LOS CARABIDAE CHILENOS}

\section{Revisiones sistemáticas}

De los 95 géneros de carábidos de Chile, se han revisado sistemáticamente 74 . Todas estas revisiones están basadas en distintos tipos de caracteres morfológicos. Actualmente, en especial para especies con alta variabilidad morfológica como Ceroglossus, se están realizando estudios de cromosomas y moleculares (Galián et al. 1996).

\section{Ecología}

Entre los trabajos específicamente sobre ecología de Carabidae en Chile, cabe mencionar el de Niemelä (1990) en Tierra del Fuego. Existen trabajos de ecología de artrópodos del suelo realizados por Elgueta (1993), que incluyen a los carábidos.

\section{Biología}

El conocimiento sobre estados inmaduros, ciclos de vida y condiciones del hábitat es realtivamente escaso, existiendo información para pocas especies (van Emdem 1942, Cekalovick 1981, Cekalovic \& Reyes 1985, Präser \& Arndt 1995). Esto no es extraño, pues para la fauna de Carabidae de América del Norte, mucho más estudiada que la de Chile, se conocen larvas de sólo 248 especies de las 2.500 descritas hasta el presente (Thompson 1979).

\section{Biogeografía}

La familia Carabidae ha sido muy utilizada para realizar inferencias biogeográficas, como las de Darlington (1965) y Jeannel (1942a). Según este último autor, las tribus de Carabidae pueden ser agrupadas de acuerdo a su origen en grandes linajes que ocupan generalmente áreas definidas. 
De estas biotas, la que posee mayor diversidad (tanto en especies como en taxa relictuales) en la Chile es la Patagónica (o Paleantártica).

Los taxa pertenecientes a la biota Patagónica se encuentran generalmente distribuidos en la región austral de América del Sur y en la región altoandina y están filogenéticamente más relacionados con otros taxa distribuidos en otras áreas australes como Australia, Tasmania, Nueva Zelanda o Africa del Sur.

\section{Estudios filogenéticos}

Muchas de las revisiones sistemáticas recientes de carábidos incluyen estudios filogenéticos. Es el caso de las tribus Bembidiini (Erwin 1974), Broscini (Roig-Juñent 1995a, 1995b, en prensa), Cnemalobini (Roig-Juñent \& Flores 1995), Lebiini (Mateu 1955, Ball \& Shpeley 1983) y Harpalini (Noonan 1981b, 1985).

\section{Museos y colecciones}

Una gran cantidad de material de Carabidae está depositado en diversas colecciones y museos de Chile. La mayor parte del material tipo de las especies descritas el siglo pasado se encuentra depositado en museos extranjeros, excepto algunas especies descritas por Phillipi, Reed y Germain, depositadas en el Museo Nacional de Historia Natural de Santiago (Camousseight 1980). Lo mismo sucede para este siglo, excepto con los materiales de especies descritas por Peña y Roig-Juñent.

La colección más completa se encuentra en el Museo Nacional de Historia Natural (Santiago). La mayoría de sus ejemplares se encuentra identificado hasta nivel de género y especie. Parte de esta colección es la de Germain. Contiene depositados materiales tipo de unas 50 especies y la mayoría de las colecciones realizadas a fines del siglo pasado, con la mayoría de los tipos descritos por R. A. Phillipi, Germain y algunos de los descritos por Jeannel. La biblioteca de este museo constituye una de las más importantes del país, con una extensa colección de revistas y trabajos del siglo XIX y del XX de éste. Otras colecciones de importancia las constituyen la del Instituto de Biología (Concepción), con una extensa colección de Carabidae, sobre todo del sur del país, la del Instituto de la Patagonia (Punta Arenas), con una de las más representativas colecciones de la Patagonia, incluyendo algunos materiales tipo de especies patagónicas.

\section{Especies amenazadas}

No se dispone de datos precisos acerca de especies en peligro de extinción. Sin embargo, muchas especies de carábidos cuya distribución es muy restringida podrían verse afectadas seriamente por alteraciones en las condiciones del hábitat.

Dos aspectos deberían desarrollarse a corto plazo. Una de las medidas a tomar, al igual que para otros grupos de artrópodos, es realizar estudios para evaluar la riqueza específica por áreas biogeográficas del país. Estos estudios han sido desarrollados en varias regiones de Chile y podría decirse que se tiene un conocimiento bastante acabado de la fauna del suelo en las distintas regiones de Chile. En la Tabla 2 se citan la cantidad de taxones (tribus, géneros y especies) para cada una de las regiones de Chile.

(1) Desierto de Atacama. Constituye la cuarta área en importancia por la cantidad de especies (Tabla 2), con un 33,7\% de ellas endémicas. Hay dos géneros endémicos. Muchas especies están sometidas a ambientes con escasos recursos y cuya alteración podría ser irreparable, como alteraciones del suelo por minería.

(2) Región central. Es el área con mayor cantidad de especies de Chile (Tabla 2), compartiendo muchas de sus especies con el desierto y la región de la Araucanía, con un 38,9\% de sus especies endémicas. Tres géneros son endémicos de esta región. Esta es una de las áreas con mayor impacto ambiental producido principalmente por la subdivisión indiscriminada de terrenos.

(3) Región de la Araucanía. Es la segunda área en importancia por la cantidad de especies (Tabla 2), con aproximadamente un $18 \%$ de especies endémicas. Posee cuatro géneros endémicos. Al igual que en la región central hay un gran impacto por la agricultura.

(4) Selva valdiviana. Esta región, al igual que la anterior, no posee una alta cantidad de endemismos (Tabla 2), pero en ella existe gran cantidad de grupos relictuales. Es la única región en donde existe una tribu endémica, la cual es compartida con los ambientes semejantes en Argentina. También en este área se encuentra la mayor cantidad de géneros endémicos.

(5) Bosque magallánico. Posee casi la mitad de las especies que están presentes en las áreas anteriores (Tabla 2). Sin embargo, existen cinco géneros endémicos y casi la mitad de sus especies también lo son. Hasta el momento, este área ha sido la menos alterada por el hombre y también es la que ha sido menos estudiada. Muchos de sus taxones son compartidos con Argentina. 
TABLA 2

Cantidad de tribus, géneros y especies para cada una de las regiones consideradas por Roig-

Juñent (1994b) para Chile. Se cita en cada columna el número de taxones presentes y los taxones endémicos. Los taxa endémicos de estas regiones en algunos casos habitan los mismos ambientes en Argentina

Number of tribes, genera, and species for each natural region adopted by Roig-Juñent (1994b) for Chile. On each columm, the first number corresponds to the number of taxa present, the second number represents the endemic taxa. Several of these endemic taxa are shared with Argentina, and they are restricted to these particular southern South American habitats

\begin{tabular}{|c|c|c|c|c|}
\hline Región & Tribus & Géneros & Especies & $\begin{array}{c}\text { Especies } \\
\text { endémicas } \\
(\%)\end{array}$ \\
\hline Desierto & $11 / 0$ & $37 / 2$ & $83 / 28$ & 33,7 \\
\hline Región central & $17 / 0$ & $53 / 3$ & $149 / 58$ & 38,9 \\
\hline Araucanía & $17 / 0$ & $47 / 4$ & $110 / 20$ & 18,1 \\
\hline Selva valdiviana & $19 / 1$ & $48 / 6$ & $99 / 28$ & 28,2 \\
\hline Bosque magallánico & $11 / 0$ & $30 / 5$ & $49 / 24$ & 48,9 \\
\hline Estepa patagónica & $7 / 0$ & $11 / 2$ & $21 / 14$ & 66,6 \\
\hline
\end{tabular}

(6) Estepa patagónica. Es el área que menor diversidad presenta en Chile, en cantidad de taxones (Tabla 2). Sin embargo, hay que hacer notar que la superficie que ocupa es muy pequeña, restringida a algunos sectores de Coyhaique y a parte de la provincia magallánica. A pesar de ello muestra dos géneros endémicos y el 66,6\% de sus especies sólo existen en este área.

\section{CONCLUSIONES}

El incremento de especies conocidas en Chile en 172 años de investigación ha subido de cinco a 365 especies. Si bien su número es inferior al de otros países de América del Sur, la cantidad de especies y géneros endémicos que posee convierte a Chile en el país de América Latina con mayor grado de endemismo.

Esto se debe a la condición insular de Chile dentro de América del Sur. Las barreras geográficas que determinan esta condición son, por el Norte, el desierto y, por el oeste, las altas cumbres de la Cordillera de los Andes. Estas dos barreras aíslan a Chile de la fauna Neotropical de Carabidae, razón por la cual podría explicarse la ausencia de Scaritini, Galeritini y Brachinini. El único sector chileno que posee contactos que permiten la difusión de su fauna se encuentra en la región austral. En esta región existe un amplio o casi exclusivo predominio de grupos patagónicos (o paleantárticos). En Carabidae dan prueba de este contacto los numerosos géneros compartidos entre Chile y Argentina (y en algunos casos con Uruguay). La mayoría de estos géneros poseen pocas especies; sin embargo, constituyen tipos morfológicos muy diferentes del resto del Neotrópico y constituyen grupos relictuales relacionados con otros taxones australes, como Broscini, Migadopini y Zolini. Existen también grupos relictuales de origen Pangéico, como Systolosomini, Ceroglossini, Cnemalobini y Omina.

El conocimiento taxonómico de Carabidae en Chile es adecuado; sin embargo, es necesaria la confección de catálogos actualizados. Para ello, es imprescindible estudiar exhaustivamente las ricas colecciones existentes en el país y otras del extranjero y la realización de viajes de colecta a áreas deficientemente conocidas.

\section{AGRADECIMIENTOS}

Expreso mi agradecimiento al Lic. Mario Elgueta y a la Dra. Esperanza Cerdeño por la lectura crítica del manuscrito.

\section{LITERATURA CITADA}

ALLEN RT (1979) The occurrence and importance of Ground beetles in Agricultural and sourrounding habitats. En: Erwin TL, GE Ball \& DR Whitehead (eds) Carabid beetles, their evolution, natural history, and classification: 485-505. Dr. W. Junk, The Hague, The Netherlands.

BALAZUC J (1957) Les Céroglosses. Revue Française d' Entomologie 24: 213-231.

BALL GE (1975) Pericaline Lebiini: Notes on classification. A synopsis of the new world genera, and a revision of the genus Phloeoxena Chaudoir (Coleoptera: Carabidae). Quaestiones Entomologicae 11: 143-242. 
BALL GE (1979a) Conspectus of carabid classification: history, holomorphology, and higer taxa. En: Erwin TL, GE Ball \& DR Whitehead (eds) Carabid beetles, their evolution, natural history, and classification: 63-111. Dr. W. Junk, The Hague, The Netherlands.

BALL GE (1979b) Epilogue. En: Erwin TL, GE Ball \& DR Whitehead (eds) Carabid beetles, their evolution, natural history, and classification: 599-618. Dr. W. Junk The Hague, The Netherlands.

BALL GE (1996) Vignettes of the history of neotropical carabidology. Annales Zoologici Fennici 33: 5-16.

BALL GE \& D SHPELEY (1983) The species of Eucheiloid pericalina: classification and evolutionary considerations (Coleoptera: Carabidae: Lebiini). Canadian Entomologist 115: 743-806.

BLACKWELDER RE (1944) Checklist of the coleopterous insects of Mexico, Central America, The West Indies, and South America. Part 1. Bulletin of the United States National Museum 185: 1-188.

BONNIARD DE SALUDO P (1969) Nouveaux carabiques du Chili. Bulletin de la Societé d'Histoire Naturelle Toulouse 105: 311-328.

CAMOUSSEIGHT A (1980) Catálogo de los tipos de insecta depositados en la colección del Museo Nacional de Historia Natural (Santiago, Chile). Publicaciones Ocasionales del Museo Nacional de Historia Natural (Chile) 32: 1-45.

CEKALOVIC T (1981) Descripción de la larva de y pupa de Ceroglossus suturalis (Fabr.), 1775 (Coleoptera, Carabidae). Boletín de la Sociedad de Biología de Concepción (Chile) 51: 67-73.

CEKALOVIC T \& M REYES (1985) Descripción de la larva de Cicindela (Plectographa) gormazi (Red, 1871) (Coleoptera, Cicindelidae). Boletín de la Sociedad de Biología de Concepción (Chile) 56: 225-229.

CHAUDOIR M (1854) Memoire sur la famille des Carabiques. Bulletin de la Société Impériale des Naturalistes de Moscou 29: 279-352.

CHAUDOIR M (1861) Materiaux pour servir a l'etude des Cicindelètes et des Carabiques. Bulletín de la Société Impérial de Naturalistes de Moscou 34: 491-576.

CSIKI E (1927-1933) Coleoptera, Carabidae. En: Junk W \& S Schenkling (eds) Coleopterorum catalogus, pars 91, Carabinae I: 1-314 (1927); pars 92, Carabinae II: 315622 (1927); pars 97, Mormolycinae, Harpalina I: 1-226 (1928); pars 98, Harpalinae II: 227-345 (1928); pars 104, Harpalinae III: 347-527 (1929); pars 112, Harpalinae IV: 529-737 (1930); pars 115, Harpalinae V: 739-1022 (1931); pars 121, Harpalinae VI: 1023-1278 (1932); pars 124, Harpalinae VII: 1279-1598 (1932); pars 126, Harpalinae VIII: 1599-1933 (1933); pars 127, Carabinae III: 623-648. W. Junk, Berlin, Alemania.

DARLINGTON PJ (1965) Biogeography of the southern end of the world. Distribution and history of the far southern life and land with assesment of continental drift. Harvard University Press, Cambridge, Massachusetts. 236 pp.

DEJEAN P (1825-1831) Spècies génèral des Coléoptères de la collection de M. Le Compte Dejean. Mequignon, París. (Vol. 1, 1825: 1-463; Vol. 2, 1826: 1-501; Vol. 3, 1828: 1-556; Vol. 4, 1829: 1-520; vol. 5, 1831: 1-883)

DEUVE T (1989) Diagnose d'une nouvelle espéce du genre Ceroglossus (Col. Carabidae). L'Entomologiste 45: 57.
ELGUETA M (1993) Invertebrados asociados a suelo en Bosque de Nothofagus pumilio (Poepp et Endl) Krasser, XII región- Chile, con especial referencia a insecta. Revista Chilena de Entomología 20: 49-60.

ELGUETA M \& G ARRIAGADA (1989) Estado actual del conocimiento de los coleópteros de Chile (Insecta: Coleoptera). Revista Chilena de Entomología 17: 5-60.

ENDERLEIN G (1912) Die Insekten des Antarkto-ArchiplataGebietes (Fuerland, Falklands Inseln, Sud Georgien). Kung. Svenska Vetenskapsakademiens Handlingar 48: 1-170.

ERWIN TL (1972) Two new genera of Bembidiine carabid beetles from Australia and South America with notes on their phylogenetic and zoogeographic significance (Coleoptera). Breviora 383: 1-19.

ERWIN TL (1974) Studies of the subtribe Tachyina (Coleoptera: Carabidae: Bembidiini): a revision of the New World-Australian genus Pericompsus LeConte. Smithsonian Contributions to Zoology 162: 1-96.

ERWIN TL (1978) Studies of the subtribe Tachyina (Coleoptera: Carabidae: Bembidiini), Supplement C: discovery of the sister group of Mioptachys Bates, in the Amazon Basin-Inpa psydroides a new genus and species. Coleopterist Bulletin 32: 29-36.

ERWIN TL (1979) Thoughts on the evolutionary history of ground beetles: hypoteses generated from comparative faunal analyses of lowland forest sites in temperate and tropical regions. En: Erwin TL, GE Ball \& DR Whitehead (eds) Carabid beetles, their evolution, natural history, and classification: 539-592. Dr. W. Junk Publishers, The Hague, The Netherlands.

ERWIN TL (1985) The taxon pulse: a general pattern of lineage radiation and extinction among carabid beetles. En: Ball GE (ed) Taxonomy, phylogeny and biogeography of beetles and ants: 437-488. Dr. W. Junk Publisher, The Hague, The Netherlands.

ERWIN TL \& LL SIMS (1984) Carabid beetles of the West Indies (Insecta: Coleoptera): a synopsis of the genera and checklist of Caraboidea, and of the West Indian species. Quaestiones Entomologicae 20: 351-466.

EVANS MEG (1980) The phylogenetic evidence of functional characters in ground beetles (Coleoptera: Caraboidea). Entomologicae Generalis 6: 303-310.

GALIÁN J, F PRÄSER, P DE LA RÚA, J SERRANO \& D MOSSAKOWSKI (1996) Cytological and molecular differences in the Ceroglossus chilensis complex (Coleoptera: Carabidae). Annales Zoologici Fennici 33: 23-30.

GEMMINGER M \& E VON HAROLD (1868) Catalogous coleopterorum huscusque descriptorum synonymiscus et systematicus. Sumptu E.H. Gummi, Mónaco, Mónaco. 424 pp.

GERMAIN P (1855a) Descripción de coleópteros de diversas especies que no se hallan en la obra del Señor Gay. Anales de la Universidad de Chile 12: 386-407.

GERMAIN P (1855b) Apuntes sobre los insectos de Chile: los Carabus chilenos. Anales de la Universidad de Chile 90: 627-683.

GERMAIN P (1893) Nouvelles notes sur les coléoptères du Chili. Actas Société Scientífique du Chili 3: 47-64.

GERMAIN P (1901) Apuntes entomolójicos. Datos sobre el jénero Cnemalobus. Anales de la Universidad de Chile: 108: 175-192. 
GERMAIN P (1905) Apuntes entomolójicos. Bembidios chilenos. Anales de la Universidad de Chile 117: 589-653.

GERMAIN P (1911) Catálogo de los coleópteros chilenos del Museo Nacional. Boletín del Museo Nacional de Historia Natural (Chile) 3: 47-73.

GIDASPOW T (1963) The genus Calosoma in central America, The Antilles, and South America (Coleoptera, Carabidae). Bulletin of the American Museum of Natural History 124: 279-312.

GUÉRIN-MÉNÉVILLE MFE (1838) Insectes du voyage de la favorite. Magazine du Zoologie 8: 225-238.

HENNIG W (1981) Insect phylogeny. John Wiley \& Sons, New York, New York. 512 pp.

JEANNEL R (1938) Les Migadopides (Coleoptera, Adephaga), une lignée subantarctique. Revue Française d'Entomologie 5: 1-55.

JEANNEL R (1941) Coléoptères Carabiques Première partie. En: Chopard L (ed) Faune France 39: 1-571. Lechevalier et Fils, Paris, Francia.

JEANNEL R (1942a) La Genése des faunes terrestres. Eléments de biogeográfie. Press Universitaries de France, Paris, Francia. 513 pp.

JEANNEL R (1942b) Coléoptères Carabiques. Deuxième partie. En: Chopard L (ed) Faune France: 573-1173. Lechevalier et Fils, Paris, Francia.

JEANNEL R (1962) Les Trechidae de la paleantarctide occidentale. En: Debouteville CD \& E Rapoport (eds) Biologie d'Amerique australe, tome 1: 527-655. Centre National de la Recherche Scientifique et Consejo Nacional de Investigaciones Científicas y Técnicas, Paris, Francia.

JEANNEL R \& R PAULIAN (1944) Morphologie abdominale des Coléoptères et systematique de l'ordre. Revue Française d'Entomologie 11: 65-110.

KAVANAUGH DH (1979a) Investigations on present climatic refugia in North America through studies on the distributions of carabid beetles: concepts, methodology and prospectus. En: Erwin TL, GE Ball \& DR Whitehead (eds) Carabid beetles, their evolution, natural history, and classification: 369-381. Dr. W. Junk, The Hague, The Netherlands.

KAVANAUGH DH (1979b) Rates of taxonomically significant differentiation to geographical isolation and habitat: examples from study of the neartic Nebria fauna. En: Erwin TL, GE Ball \& DR Whitehead (eds) Carabid beetles, their evolution, natural history, and classification: 35-57. Dr. W. Junk, The Hague, The Netherlands.

KOLBE H (1907) Hamburger Magalhaensische Sammelreise Coleopteren. Mit 3 karten: 1-125. L. Friederichsen \& Co., Hamburgo, Alemania.

LACORDAIRE T (1854) Histoire naturelle des insectes: genera des coléoptères, volumen I. Libraire Encyclopédique de Roret, Paris, Francia. 486 pp.

LAGOS S \& S ROIG-JUÑENT (1997) Una nueva especie de Cnemalobus de Chile (Coleoptera: Carabidae). Revista Chilena de Entomología 24: 27-30.

LIEBHERR JK (1994) Identification of New World Agonum: review of the mexican fauna, and description of Incagonum new genus, form South America (Coleoptera: Carabidae: Platyinini). Journal of the New York Entomological Society 10: 1-55.
LIEBKE M (1931) Laufkäfer studien VIII. Neue gattungen und arten der unterfamilie Ctenodactyline. Entomologische Anzeiger 11: 358-361, 389-392.

LIEBKE M (1935) Neue carabiden aus Süd-und Mittelamerïka, hauptsächlich des pariser museums. Revue Française d'Entomologie 2: 143-177.

MATEU J (1954) El género Callidula Chaudoir. Revista de la Sociedad Entomológica Argentina 17: 3-8.

MATEU J (1955) Revisión de los géneros Mimodromius Chaudoir y Mimodromites Mateu (Coleoptera: Carabidae). Archivos del Instituto de Aclimatación 4: 63-108.

MATEU J (1959) Nuevos datos sobre Mimodromius Chaudoir (Coleoptera: Carabidae). Doriana 3: 1-11.

MATEU J \& N NÈGRE (1972) Révision du genre Trechisibus Motsch. et genres voisins. Nouvelle Revue d'Entomologie 2: 53-72.

MOORE BP (1979) Chemical defense in carabids and its bearing on phylogeny. En: Erwin TL, GE Ball \& DR Whitehead (eds.) Carabid beetles, their evolution, natural history, and classification: 193-203. Dr. W. Junk, The Hague, The Netherlands.

NÈGRE J (1963) Révision du genre Polpochila Solier (Col. Carabidae). Revue Française d'Entomologie 30: 205241.

NÈGRE J (1973) The zoological results of Gy. Tópal's collectings in South Argentine. 24. Coléoptères: Carabidae. Folia Entomologica Hungarica 26: 289-310.

NIEMELÄ J (1990) Habitat distribution of carabid beetles in Tierra del Fuego, South America. Entomologia Fennica 1: 3-16.

NIEMELÄ J (1996) From systematics to conservationcarabidologist do it. Annales Zoologici Fennici 33: 1-4.

NOONAN GR (1974) Allendia, a new South American genus with notes on its evolutionary relationships to other genera of Anisodactylina (Carabidae: Coleoptera: Harpalini). Coleopterist Bullletin 28: 219-227.

NOONAN GR (1976) Synopsis of the supra-specific taxa of the tribe Harpalini (Coleoptera: Carabidae). Quaestiones Entomologicae 12: 3-87.

NOONAN GR (1979) The science of biogeography with relation to carabids. En: Erwin TL, GE Ball \& DR Whitehead (eds) Carabid beetles, their evolution, natural history, and classification: 295-317. Dr. W. Junk, The Hague, The Netherlands.

NOONAN GR (1981a) South American species of the subgenus Anisotarsus Chaudoir (genus Notiobia Perty: Carabidae: Coleoptera), part I: taxonomy and natural history. Milwaukee Public Museum 44: 1-84.

NOONAN GR (1981b) South American species of the subgenus Anisotarsus Chaudoir (genus Notiobia Perty: Carabidae: Coleoptera), part II: evolution and biogeography. Milwaukee Public Museum 45: 1-117.

NOONAN GR (1985) Reconstructed phylogeny and zoogeography of the genera and subgenera of the Selenophori group (Insecta: Coleoptera: Carabidae: Harpalini: Harpalina). Milwaukee Public Museum 65: 1-33.

PEÑA LE \& G BARRÍA (1973) Revisión de la familia Cicindelidae (Coleoptera), en Chile. Revista Chilena de Entomología 7: 183-191.

PHILIIPPI RA (1862) Sobre algunos insectos de Magallanes. Anales de la Universidad de Chile 1862: 407-414.

PHILIPPI RA (1887) Catálogo de los coleópteros de Chile. Anales de la Universidad de Chile 71: 659-806. 
PHILIPPI RA \& C JULIET (1871) Esploración científica practicada por órden del Supremo Gobierno i segun las instrucciones del Doctor don R. A. Philippi, por don Carlos Juliet, ayudante de la Comision exploradora del mar i costas de Chiloé i Llanquihue, a bordo del Covandonga. Anales de la Universidad de Chile 39: 81168.

PRÄSER F \& E ARNDT (1995) Description of the larva of Ceroglossus chilensis Eschscholtz, 1829 (Insecta: Coleoptera). Studies on Neotropical Fauna and Environment 30: 229-235.

REED C (1874) On the Coleoptera Geodephaga of Chile. Proceedings of the Zoological Society of London 1874: 48-70.

REICHARDT H (1977) A synopsis of the genera of neotropical Carabidae (Insecta: Coleoptera). Quaestiones Entomologicae 13: 346-393.

REICHARDT H (1979) The South American carabid fauna: endemic tribes and tribes with African relationships. En: Erwin TL, GE Ball \& DR Whitehead (eds) Carabid beetles, their evolution, natural history, and classification: 319-325. The Hague. Dr. W. Junk, The Netherlands.

ROIG-JUÑENT S (1992) Revisión del género Barypus Dejean, 1828 (Coleoptera: Carabidae: Broscini) Parte II. Revista de la Sociedad Entomológica Argentina 50: 89-117.

ROIG-JUÑENT S (1993) Cnemalobini, una tribu de Carabidae (Coleoptera) endémica de América del Sur. Acta Entomológica Chilena 18: 7-18.

ROIG-JUÑENT S (1994a) Las especies chilenas de Cnemalobus Guérin-Ménéville, 1838 (Coleoptera: Carabidae: Cnemalobini). Revista Chilena de Entomología 21: 5-30.

ROIG-JUÑENT S (1994b) Historia biogeográfica de América del Sur austral. Multequina 3: 167-203.

ROIG-JUÑENT S (1995a) Cladistic analysis of Barypus Dejean 1828 (Coleoptera: Carabidae: Broscini). American Museum Novitates 3117: 1-11.

ROIG-JUÑENT S (1995b) Revisión sistemática de los Creobiina de América del Sur (Coleoptera: Carabidae: Broscini). Acta Entomológica Chilena 19: 51-74.

ROIG-JUÑENT S (1998a) Carabidae. En: Morrone JJ \& S Coscarón (eds) Biodiversidad de artrópodos argentinos, un enfoque biotaxonómico: 194-209. Ediciones Sur, La Plata, Argentina.

ROIG-JUÑENT S (1998b) Cladistic relationships of the tribe Broscini (Coleoptera: Carabidae). Atti delle Museo Regionale di Scienze Naturali di Torino (Italia) 1998: 343-358.

ROIG-JUÑENT S (2000) The subtribes and genera of the tribe Broscini (Coleoptera: Carabidae): cladistic analysis, taxonomic treatment, and biogeographical considerations. Bulletin of the American Museum 255: $1-98$.

ROIG-JUÑENT S \& A CICCHINO (1989) Revisión del genero Barypus Dejean, 1828 (Coleoptera: Carabidae: Broscini), parte I. Boletín de la Sociedad de Biología de Concepción (Chile) 60: 201-225.
ROIG-JUÑENT S \& A CICCHINO (en prensa) A new genus and species belonging to a new subtribe of Zolini (coleoptera: carabidae) from Southern South America. Canadian Entomologist.

ROIG-JUÑENT S \& GE FLORES (1995) Análisis cladístico del género Cnemalobus (Coleoptera: Carabidae: Cnemalobini). Boletín de la Sociedad de Biología de Concepción (Chile) 66: 155-168.

RUÍZ-PEREIRA F (1937) Los Ceroglossus de Chile (Col. Carabidae). Revista Chilena de Historia Natural 40: 381 425.

SHPELEY D (1986) Genera of the subtribe Metallicina and classification, reconstructed phylogeny and geographical history of the species of Euproctinus Leng and Mutchler (Coleoptera: Carabidae: Lebiini). Quaestiones Entomologicae 22: 261-349.

SLOANE TG (1923) The classification of the family Carabidae. Transanctions of the Entomological Society of London 1923: 234-250.

SOLIER AJJ (1849) Ordre III. Coléoptères. En: Gay C (ed) Historia Física y Política de Chile, volumen 4: 105-380, 414-511. Fain \& Thunot, París, Francia.

STRANEO SL (1951) Sur la tribu des Metiini (Antarctiini aust.) (Coleoptera Pterostichidae). Revue Française d’Entomologie 18: 56-88.

STRANEO SL (1958) Su alcuni Carabidi (Coleoptera). Atti delle Societa Italiana Science Naturali 97: 37-40.

STRANEO SL (1963) Un interessante carabide raccolto dal D. Kuschel nell'isolla di S. Ambrosio (America Meridionale). Revue Françacise d'Entomologie 30: 124127.

STRANEO SL (1969) Sui carabidi del Chile, raccolti dal Dr. Holgate della Royal Society expedition (1958-1959) e dal Prof. Kuschel. Annales de la Société Entomologique de France (NS) 5: 951-971.

STRANEO SL (1986) Sul genre Blennidius Mostchulsky 1865 (Col. Carabidae, Pterostichini). Bolletino delle Museo Regionale di Scienze Naturali di Torino (Italia) 4: 369-393.

STRANEO SL (1995) Sul genere Feroniola Tschitscherine (Coleoptera Carabidae). Atti delle Societa Italiana Science Naturali 134: 17-14.

STRANEO SL \& R JEANNEL (1955) Los insectos de las islas Juan Fernandez. 23. Carabidae (Coleoptera). Revista Chilena de Entomología 4: 121-144.

THIELE HU (1977) Carabid beetles in their environments: a study on habitat selection by adaptions in physiology and behaviour. Springler-Verlag, Berlin, Alemania. 360 pp.

THOMPSON RG (1979) Larvae of North American Carabidae with a key to the tribes. En: Erwin TL, GE Ball \& DR Whitehead (eds) Carabid beetles, their evolution, natural history, and classification: 209-291. Dr. W. Junk, The Hague, The Netherlands.

VAN EMDEN FI (1942) A key of the genera of larval Carabidae (Col.). Transactions of the Royal Entomological Society of London 92: 1-99.

VAN EMDEM FI (1953) XLIX-The Harpalini genus Anisotarsus Dejean (Col. Carab.). Annals and Magazine of Natural History 6: 513-547. 


\section{ANEXO 1 \\ Claves para la identificación de los géneros de Carabidae de Chile y notas acerca de cada género presente en Chile}

\section{La organización de las claves es la siguiente: se da una clave de las tribus de Carabidae de Chile y luego para cada tribu, cuando existan más de dos géneros, se proporciona una clave para su identificación}

Clave para la identificación de las tribus de Carabidae chilenas

1- Clípeo más ancho que la distancia entre las inserciones antenales. .. (Carabinae parte ................................. 2

1'- Clípeo más angosto que la distancia entre las inserciones antenales ........................................................... 3

2- Pronoto con los ángulos anteriores prominentes; palpómero maxilar 4 más corto que el 3; cuerpo sin pubescencia Megacephalini

2’- Pronoto con los ángulos anteriores no prominentes; palpómero maxilar 4 más largo que el 3; cuerpo con pubescencia.

Cicindelini

3- Tibia anterior con las espinas terminales independientes del órgano de limpieza

3'- Tibia anterior con una espina terminal y la otra asociada al órgano de limpieza .....

4- Coxa posterior extendida lateralmente hasta la epipleura elitral ....... Trachypachinae ............................................

4'- Coxa posterior no extendida, no contactando la epipleura elitral ..... Paussinae ....................................... Ozaenini

5- Cavidades coxales anteriores abiertas posteriormente .................... Carabinae parte ................................... 6

5' - Cavidades coxales anteriores cerradas posteriormente …....................................................................... 7

6- Antenómeros 2 y 3 carenados; mandíbulas al menos basalmente con rugosidades transversas; labro negro ........ Carabini

6' - Antenómeros 2 y 3 cilíndricos, no carenados; mandíbulas lisas o finamente punteadas; labro metálico Ceroglossini

7- Cavidades coxales medias disyuntas (no cerradas enteramente por el esterno); estría escutelar extendida hasta el ápice, paralela a la sutura elitral Migadopini

7'- Cavidades coxales medias conjuntas (cerradas enteramente por el esterno); estría escutelar ausente o presente, pero

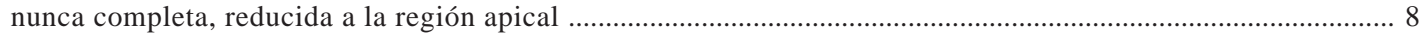

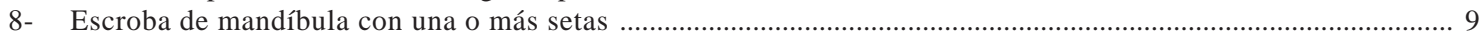

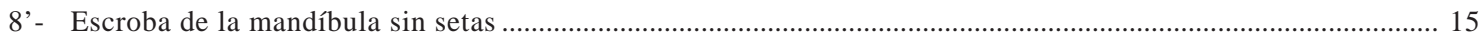

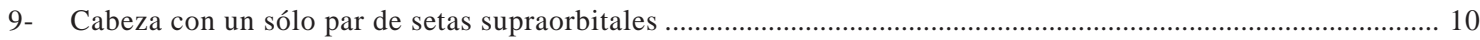

9'- Cabeza con más de un par de setas supraorbitales .................................................................................. 11

10- Último palpómero maxilar tan o más largo que el anterior ............... Broscini (parte)

10' - Último palpómero maxilar muy pequeño, casi escondido dentro del anterior .....

1- Cabeza con tres o más pares de setas supraorbitales Bembidiini (parte)

.......... Broscini (parte)

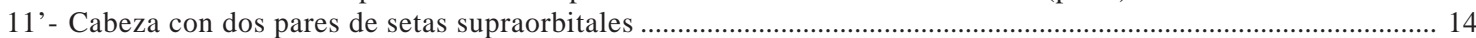

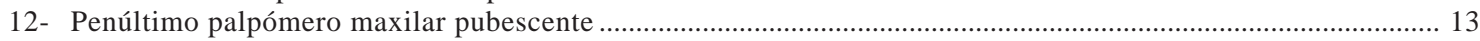

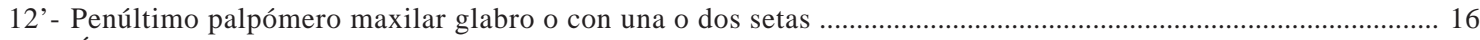

13- Último palpomero maxilar más corto y delgado que el penúltimo; élitro con base marginada Bembidini

13' - Último palpómero maxilar tan largo como el anterior; base del élitro no marginada Zolini

14- Surcos frontales curvados al medio, llegando hasta atrás de los ojos; distancia entre el ojo y el surco adyacente subigual a la distancia entre los surcos; esclerito glosal con seis o más setas .....

\section{Trechini}

14'- Surcos frontales poco curvados o rectos, cortos, no sobrepasando la mitad del ojo; en el medio más distantes uno de otro que la distancia con el ojo; esclerito glosal con dos o tres setas

$$
\text { Psydrini }
$$

15- Cabeza con tres o más pares de setas supraorbitales; tibia anterior extendida lateroapicalmente en un diente .....

15 ' - Cabeza con uno o dos pares de setas supraorbitales Cnemalobini (parte)

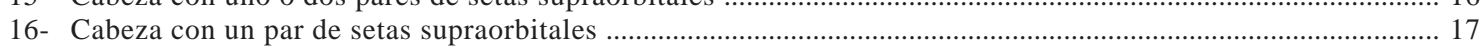

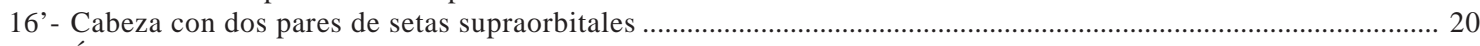

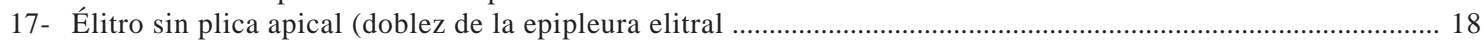

17 ' - Élitro con plica apical; superficie del élitro y pronoto fina y densamente punteada.

Chlaenini

18- Antenómero 3 con pocas setas, no pubescente, 4 pubescente en el tercio apical 
18'- Antenómero 3 pubescente en el tercio apical, 4 completamente pubescente

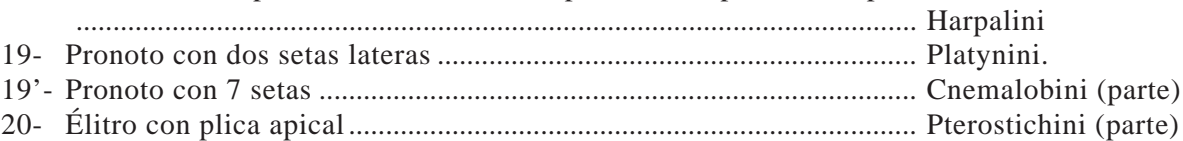

20' - Élitro sin plica apical

21- Pronoto angosto, más largo que ancho, borde posterior tan ancho como el anterior. Tarsómero 4 profundamente hendido en el ápice, bilobado casi hasta la mitad Ctenodactylini

21' - Pronoto no más largo que ancho, borde posterior más angosto que el anterior

22- Longitud de la espina interna de la tibia posterior más larga que la mitad del basitarso posterior, espina interna mayor que la externa. Masoreini

22'- Longitud de la espina interna de las tibias posteriores menor que la mitad del largo del basitarso; ambas espinas subiguales

23- Cabeza con un par de macrosetas ventrales posteriores al submentón

23' - Cabeza sin par de macrosetas posteriores al submentón Lebiini (parte)

24- Élitro con el margen apical truncado ...................................................... Lebiini

24 ' - Élitro con el margen apical no truncado, sinuado o no......

25-Clípeo hundido basalmente, superficie más o menos cóncava, emarginado anteriormente; labro profundamente truncado Licinini

25' - Clípeo plano, no hundido, margen anterior recto o levemente emarginado; labro con borde anterior truncado o levemente cóncavo Platynini

\section{TRACHYPACHINAE SYSTOLOSOMINI}

(1) Systolosoma Solier, 1849 Es un género endémico de los bosques de Nothofagus, en la Selva Valdiviana. Su grupo hermano lo constituye la tribu Trachypachini de los bosques costeros meridionales de América del Norte. Muchos consideran a este grupo como un Hydradephaga, mientras que otros lo consideran como el grupo hermano de Carabidae (Erwin 1985), con rango de familia o subfamilia. En Chile se encuentran las dos especies del género (Solier 1849, Nègre 1973).

\section{CARABINAE}

CARABINI

(2) Calosoma Weber, 1801. Género de distribución mundial. Para América del Sur, la revisión más completa y reciente es la de Gidaspow (1963). Para el Neotrópico, hay 61 especies de las cuales sólo hay presentes cuatro en Chile, pertenecientes a dos subgéneros.

\section{CEROGLOSSINI}

(3) Ceroglossus Solier, 1849. Género restringido a los bosques de Nothofagus, en toda su extensión. Posee ocho especies (cinco de ellas endémicas de Chile). Elgueta \& Arriagada (1989) consideran que la especie de Deuve (1989) sería un sinónimo, sin embargo aquí en este trabajo se considera como válida hasta que no sea sinonimizada. Las especies de Ceroglossus han llamado la atención de los naturalistas desde que se empezó a colectar en Chile, llegando a proponerse hasta 120 nombres como válidos. Con la revisión de Balazuc (1957) quedó establecido claramente que la cantidad de especies era mucho menor. Galián et al. (1996) hicieron un estudio citológico y molecular del grupo chilensis.

\section{CICINDELINI}

(4) Cicindela Linneus, 1758. Género con distribución mundial, con alrededor de 700 especies, 117 en la región Neotropical. Para Chile, la revisión más completa es la de Peña \& Barría-Pereira (1973). Las cinco especies presentes en Chile se encuentran distribuidas desde Atacama hasta la Selva Valdiviana. Una de ellas, Cicindela (Plectographa) nahuelbutae (Peña \& Barría-Pereira 1973), endémica de la región de la Araucanía.

\section{MEGACEPHALINI}

1- Élitro sin húmero, con pseudohúmero y pseudoepipleuron, cuyo ángulo superior forma una carena. Superficie dorsal del élitro con carenas. Coxas posteriores separadas. Omina Pycnochila

1'- Élitro con el húmero de diversa forma, escondido bajo el pseudohúmero; pseudoepipleuron ausente. Superficie dorsal del élitro lisa, sin carenas. Coxas posteriores tocandose en la región sagital

$$
\text { Megacephalina }
$$

(5) Megacephala Latreille, 1802. Género de distribución mundial con aproximadamente 40 especies Neotropicales. Para Chile hay dos especies. Una citada para Arica y La Serena y otra para Caupolicán. 
ANEXO 1 (continuación)

(6) Pycnochila Motschulsky, 1856. Género monoespecífico, Pycnochila fallaciosa (Chevrolat, 1854), que habita en regiones de estepa del Estrecho de Magallanes. Está estrechamente relacionado con taxones que habitan el oeste de América del Norte.

\section{MIGADOPINI}

1- Galea maxilar biarticulada; mentón y submentón separados; glosa sin paraglosas; sin cuello definido; élitros sin trazas de setas distales

Migadopina .....

1- Galea maxilar uniarticulada; mentón fusionado al submentón; glosa con paraglosas membranosas; cabeza con cuello definido; élitros con una seta sobre el cuarto basal de la tercera estría

Monolobina Monolobus

2- Diente del mentón bilobulado 3

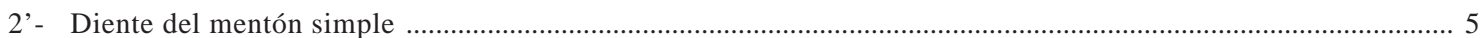

3- Mesotarsos de los machos simples, ni dilatados ni revestidos de setas adhesivas; tibias anteriores muy dilatadas en los machos; esclerito glosal unisetoso.... Lissopterus

3' - Mesotarsos de los machos dilatados o no, pero siempre con órganos adhesivos ventrales; esclerito glosal uni o

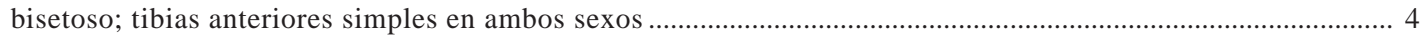

4- Esclerito glosal bisetoso; apófisis prosternal prolongada hacia atrás y apoyada en el mesosterno; pronoto transverso, con ángulos posteriores casi rectos; élitros oblongos, con estrías superficiales, finas, borradas hacia fuera; serie umbilical con 15 a 20 setas ..... Migadops

4'- Esclerito glosal unisetoso; apófisis prosternal de forma normal; pronoto no transverso, subcordiforme, con los ángulos posteriores muy agudos, élitros cortos, con las estrías marcadas por gruesos puntos, serie umbilical con pocas setas Pseudomigadops

5- Coloración parduzca, no metálica; pronoto transverso, con margen estrecho y regular, élitros con estrías enteras; protarsómero 4 bilobulado ..... Antarctonomus

5'- Coloración metálica, azulada o verdosa, brillante, con una mancha subapical redondeada de color testácea en cada élitro; pronoto no transverso, con un margen muy ancho, élitros lisos. protarsómero 4 no bilobadoMigadopidius

(7) Antarctonomus Chaudoir, 1861. Género monoespecífico (Antarctonomus complanatus (Blanchard, 1853) en Argentina y Chile, distribuido en bosques subantárticos desde Chiloé hasta Tierra del Fuego. Revisión de Jeannel (1938).

(8) Lissopterus Waterhouse, 1843. Dos especies de la estepa de Magallanes e Islas Malvinas, ambas presentes en Chile. Revisión de Jeannel (1938).

(9) Migadopidius Jeannel, 1938. Género monoespecífico (Migadopidius bimaculatus (Reed, 1874), desde la región de la Araucanía hasta la provincia argentina de Santa Cruz. Revisión de Jeannel (1938).

(10) Migadops Waterhouse, 1842. Dos especies de los bosques subantárticos. Ambas presentes en Chile, una endémica. Revisión de Jeannel (1938).

(11) Monolobus Solier, 1849. Dos especies de la Selva Valdiviana de Chile y Argentina. Una endémica de Chile. De los bosques de Nothofagus. Revisón de Jeannel (1938).

(12) Pseudomigadops Jeannel, 1938. Cinco especies, cuatro presentes en Chile, dos endémicas. Sus especies se encuentran en regiones de estepa Patagónica. Revisión de Jeannel (1938) y Straneo (1969).

\section{PAUSSINAE}

OZAENINI

1- Mesocoxas contiguas o al menos no separadas por la unión del mesosterno con el metasterno

\section{Pachyteles}

1'-Mesocoxas separadas en el medio por la unión del meso y metasterno Tropopsis

(13) Pachyteles Perty, 1830. No hay revisión de este género y es muy difícil la identificación de sus 51 especies. Una sóla especie en Chile (Pachyteles gracilis Chaudoir, 1868) endémica de la región costera, desde Coquimbo hasta Valparaíso.

(14)Tropopsis Solier, 1849. Cinco especies, tres de Chile (una sin describir) y Argentina, una de Brasil y otra de América Central. En Chile las tres especies son de los bosques australes (Región de la Araucanía y Selva Valdiviana).

\section{PSYDRINAE}

\section{BEMBIDINI}

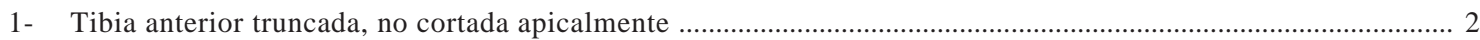

1'- Tibia anterior oblicua y fuertemente cortada lateroapicalmente .................................................................... 3

2- Estría escutelar presente, sin estría recurrente en el ápice elitral ...... Bembidiina ............................................. 7

2'- Estría escutelar ausente, con estría recurrente .................................... Xystosomina ................................optachys

3- Cuerpo pálido y generalmente piloso, con o sin ojos; si presentan ojos con la cabeza parcialmente cubierta por el pronoto

3'- Cuerpo pálido u oscuro, solo con setas táctiles, ojos siempre presentes, cabeza nunca cubierta por el pronoto Tachyna 
ANEXO 1 (continuación)

4- Labro profundamente escotado y cubriendo las mandíbulas; ápices elitrales suaves, separados en la sutura y más o menos truncados; alas y ojos presentes ......................................... Tachyna .................................. Micratopus

4'- Labro entero, no cubriendo las mandíbulas; ápices elitrales normales, no suaves, no separados y redondeados; alas y ojos siempre ausentes ............................................................ Anillina ................................. Nothanillus

5- Mentón con dos foveas profundas, circulares o con forma de herradura 6

5'- Mentón sin foveas profundas, sin impresiones o, si están presentes, son muy superficiales Elaphropus

6- Estría recurrente del élitro larga, prolongada anteriormente y curvada en forma de gancho; interestría 8 subsulcada en la mitad posterior ......................................................................... Paratachys

6'- Estría recurrente del élitro ausente o, si está presente, corta y no curvada en gancho; interestrías elitrales punctuadas o estriado sulcados ....................................................................... Pericompsus

7- Setas discales del élitro insertadas en la tercera interestría; estriación generalmente fina.......(serie Notaphus) 9

7' - Setas discales del élitro insertadas en la tercera estría ..................................................................................... 8

8- Especies convexas, con la estriación fuerte pero borrada lateralmente, las estrías punteadas; edéago con lámina derecha del orificio basal atrofiada ................................................ serie Peryphus .................................... 13

8'- Especies deprimidas, con la estriación entera, las estrías lisas; edéago con el orificio basal del lóbulo medio encuadrado dentro de láminas subiguales ......................................... Bembidarenas

9- Pronoto siempre amplio, más ancho que la cabeza, base ancha y costados sin sinuosidad posterior 10

9'- Pronoto estrecho, cordiforme, no más ancho que la cabeza, base estrecha y sus costados fuertemente sinuados.

10- Élitros con estrías fuertemente punteadas ........................................ Nothocys

10 ' - Élitros con estrías finas y superficiales, muy finamente punteadas .

11- Cuarta seta de la serie umbilical más separada de la 3 que ésta de la primera; los élitros muy comúnmente con manchas interestriales amarillas sobre cuatro fascies transversas; protarsitos 1-2 de los machos moderadamente dilatados Notaphus

11' - Setas humerales de la serie umbilical equidistantes; negros o bronceados de color uniforme, sin manchas en los élitros; protarsito 1 de los machos muy grueso e hinchado, el 2 pequeño ........................................ Notaphiellus

12- Setas humerales equidistantes; primera seta discal inserta en una profunda foseta que deprime las interestrías vecinas; tarsos anteriores de los machos con el primer artículo muy engrosado, el segundo pequeño Notholopha

12' - Cuarta seta humeral muy separada de la tercera; primera seta discal insertada sobre la tercera interestría sin ninguna depresión; tarsos anteriores del macho con el primer artículo poco engrosado............................ Pseudotrepanes

13- Élitros sin estrías, a lo sumo con trazas de la puntuación en el lugar de las dos primeras, dejando ver la inserción de la seta discal que se ubica en la tercera; tarsos anteriores de los machos con el primer artículo engrosado, el segundo pequeño ........................................................................... Nothonepha

13- Élitros con estrías bien marcadas, protarsitos 1-2 de los machos moderadamente dilatados....... 14

14- Grupo humeral de la serie umbilical con las tres primeras setas equidistantes, la cuarta separada o no de la tercera Peryphus

14' - Grupo humeral de la serie umbilicada con las setas 2 y 3 setas proximas la una de la otra, la 1 separada de la 2 y la 4 más separada de la 3

15- Surcos frontales paralelos; la estría recurrente prolongada por la estría 5 .

$$
\text { Plocamoperyphus }
$$

15'- Surcos frontales convergentes hacia delante, estría recurrente se continua con la estría 7

(15) Bembidarenas Erwin 1972. Dos especies de Chile y Argentina, de bosques de Nothofagus. Descripción de las especies por Erwin (1972) y Nègre (1973).

(16) Bembidion Latreille, 1802. Género de distribución mundial. Según Reichardt (1977), hay sólo una especie de Chile en el subgénero Chrysobracteon. La única especie que no fue tratada por Jeannel (1962) fue Bembidium chlorostictum Reed, 1874, que quizás sea la citada por Reichardt (1977).

(17) Elaphropus Mostschulsky, 1839. Género con 32 especies neotropicales; en Chile sólo una especie, Elaphrophus (Elaphrophus) yunax (Darlington, 1939), probablemente transportada por el comercio (Erwin 1978).

(18) Micratopus Casey, 1914. El género tiene cinco especies; en Chile hay citada sólo una probablemente transportada por el comercio (Erwin 1978).

(19) Mioptachys Bates, 1882. Una especie en Chile, probablemente introducida por el comercio (Erwin 1978).

(20) Nothanillus Jeannel, 1962. Género endémico de la región central de Chile. Dos especies. Insectos sin ojos y epígeos que miden muy pocos milímetros. Revisión por Jeannel (1962).

(21) Notaphiellus Jeannel, 1962. Incluye seis especies, principalmente de la Patagonia. Todas ellas presentes en Chile, tres endémicas, desde Valparaíso hasta Magallanes. Revisión por Jeannel (1962).

(22) Notaphus Stephens, 1928. Incluye 24 especies neotropicales, agrupadas en tres subgéneros propuestos por Jeannel (1962) y Bonniard de Saludo (1969). En Chile se encuentran los tres subgéneros, con 16 especies, nueve de ellas endémicas. Su distribución va desde Atacama hasta la región de Magallanes. Revisión por Jeannel (1962) y Bonniard de Saludo (1969). 
ANEXO 1 (continuación)

(23) Nothocys Jeannel, 1962. Con 11 especies de Argentina y Chile (Patagonia). En Chile hay ocho especies, seis endémicas, desde Atacama hasta Magallanes. Revisión por Jeannel (1962).

(24) Notholopha Jeannel, 1962. Incluye diez especies en su mayoría patagónicas, agrupadas en dos subgéneros. Todas ellas presentes en Chile, desde Santiago hasta Magallanes, ocho endémicas. Revisión por Jeannel (1962).

(25) Nothophena Jeannel, 1962. Dos especies distribuidas en Chile y Argentina. En Chile, desde Atacama hasta Santiago. Revisión por Jeannel (1962)

(26) Notoperyphus Bonniard de Saludo, 1969. Dos especies, endémicas de Antofagasta y Coquimbo.

(27) Paratachys Casey, 1918. Género con distribución mundial. En el Neotrópico, desde México a Argentina y Chile, con 47 especies descritas y con centenares de especies neotropicales sin describir (Reichardt 1977). Para Chile sólo una especie, Paratachys hydrophilus (Germain, 1906), de Chile central.

(28) Pericompsus LeConte, 1851. Desde los Estados Unidos a Chile y Argentina, con 59 especies agrupadas en dos subgéneros. Revisión por Erwin (1974). En Chile hay tres especies (una endémica) desde Coquimbo a Valdivia.

(29) Peryphus Stephens, 1928. Este género incluye 16 especies neotropicales, que fueron agrupadas por Jeannel (1962) en tres subgéneros, todos presentes en Chile, con 13 especies distribuidas desde Coquimo hasta Magallanes, seis de ellas endémicas.

(30) Plocamoperyphus Jeannel, 1962. Género monoespecífico de Chile y Argentina, Plocamoperyphus mandibularis (Solier, 1849), que está en la región chilena de Coquimbo.

(31) Pseudotrepanes Jeannel, 1962. Género monoespecífico, Pseudotrepanes derbesi (Solier, 1849), de la región central de Chile. Revisión por Jeannel (1962).

\section{TRECHINI}

1- Dientes de la mandíbula muy agudos, habitan la costa marina ......... Aepina ................................................. 2

1'- Dientes de la mandíbula obtusos, no habitan en la costa ................... Homaloderina .......................................... 3

2- Ultimo artículo de los palpos cónico, mentón con diente simple ...... Kenodactyllus

2'- Ultimo artículo de los palpos cilíndrico y glabro, mentón con diente bífido

3- Ojos grandes, pronoto trapezoide, deprimido, no estrechado atrás, con un surco transversal prebasal que se continúa sin interrupción por el margen lateral

3'- Ojos normales; pronoto más o menos cordiforme, estrechado en la base, con el surco transversal prebasal

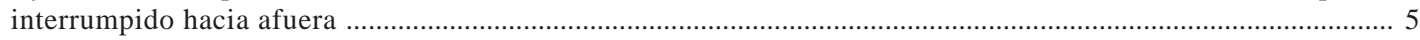

4- Pronoto transverso, con la base más ancha que el borde anterior, sin trazas de fosetas basales; élitros sin setas discales Homalodera

4'- Pronoto subcuadrado, de base casi tan ancha como el borde anterior, con fosetas basales poco profundas; élitros con tres setas discales ......................................................................... Homaloderes

5- Élitros con estría escutelar y una recurrente apical......

5' - Élitros sin estría escutelar, la primera estría profundizada y pinzada hacia adentro cerca, del escudete ............ 9

6- Estrías de los élitros siempre efuminadas hacia fuera; especies más o menos pigmentadas, con los ojos tan largos como la región temporal, tibias anteriores glabras sobre la faz anterior..

6'- Estrías externas de los élitros casi tan profundas como las internas; especies despigmentadas, con los ojos pequeños, tibias anteriores pubescentes sobre su faz anterior ........................... Putzeysius

7- Sin setas en las interestrías elitrales ................................................ Trechicomimus

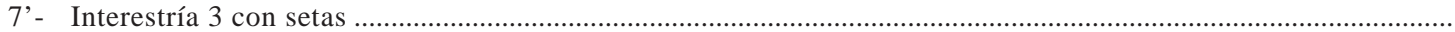

8- Una seta sobre la estría 7 y a veces también en la 2 ó 5 ..................... Nothotrechisibus

8'- Setas sólo en la interestría 3 ........................................................... Trechisibus

9- Base del pronoto rectilínea; últimos palpómeros cónicos, tan gruesos en la base como los penúltimos Pseudocnides

9'- Base del pronoto con la parte media lobulada, formando un corto pedúnculo; último palpómeros largos y glabros, subcilíndricos, más estrechos que el anterior

10- Diente del mentón bífido; élitros con una estría recurrente corta; coloración negra, con los costados del pronoto y de los élitros ampliamente testáceos Trechinotus

10'- Diente del mentón simple; élitros sin estría recurrente; coloración variable, pero nunca con un borde lateral parduzco Oxytrechus

(32) Aemalodera Solier, 1849. Género distribuido en los bosques de Nothofagus, desde Tierra del Fuego hasta Chile Central. El género posee cuatro especies, todas presentes en Chile (una endémica), distribuidas la mayoría en la Selva Valdiviana. Revisión por Jeannel (1962).

(33) Homaloderes Jeannel, 1962. Género monoespecífico (Homaloderes germaini Jeannel, 1962) endémico de Chile, de la región de la Araucanía.

(34) Kenodactyllus Broun, 1909. Género con hábitos marinos (costero) con distribución subantárctica. Dos especies, una de Auckland y otra (K. audouini [Guérin-Ménéville, 1838]) de Chile (Tierra del Fuego y Magallanes), Argentina (Tierra del Fuego y Malvinas), Islas Antípodas, Islas Auckland, Islas Campbell, Islas Snares e Islas Steward. 
ANEXO 1 (continuación)

(35) Nothotrechisibus Uéno, 1971. Género monoespecífico de Chile, Nothotrechisibus hornensis (Fairmaire, 1888), del estrecho de Magallanes.

(36) Oxytrechus Jeannel, 1927. Cuatro especies desde Ecuador a Chile y Argentina-Uruguay. Una especie endémica de Chile septentrional, Oxytrechus fasciger (Putzeys, 1870).

(37) Pseudocnides Jeannel, 1927. Género con nueve especies. Mateu \& Nègre (1972) reconocen tres subgéneros, dos de los cuales están presentes en Chile, con cinco especies, cuatro endémicas, distribuidas desde Atacama hasta Tierra del Fuego.

(38) Putzeysius Jeannel, 1962. Monoespecífico, Putzeysius quadriceps (Putzeys, 1870), endémico de Chile, probablemente de la región Norte (Pampas de Chile).

(39) Thalassobius Solier, 1849. Género monoespecífico, Thalassobius testaceus Solier, 1849, endémico de Chile de hábitos marinos (costeros) en la región de Valdivia y Chiloé.

(40) Trechichomimus Mateu \& Nègre, 1972. Género monoespecífico, Trechichomimus aphaenops Mateu \& Nègre, 1972, endémico del Sur de Chile

(41) Trechinotus Jeannel, 1962. Género endémico de Chile con tres especies de Magallanes y Selva Valdiviana.

(42) Trechisibus Motschulsky, 1862. Género con distribución adina. Revisiones por Nel (1962), Bonniard de Saludo (1969) y Mateu \& Nègre (1972). Está constituido por seis subgéneros. Incluye 37 especies presentes en Chile, 28 de las cuales son endémicas.

\section{ZOLINI}

(43) Merizodus Solier, 1849. Adultos ápteros. Cuatro especies, de las cuales tres están presentes en Chile, una endémica. Desde Chile central hasta Magallanes. Revisión por Jeannel (1962).

\section{PSYDRINI}

1- Base del élitro marginada.

Melisoderina

Tropopterus

1'- Base del élitro no marginada Meonidina Bembidiomorphum

(44) Bembidiomorphum Champion, 1918. Género monoespecífico de Chile, Bembidiomorphum convexum Champion, 1918, del Bosque Magallánico (Puerto Edén).

(45) Tropopterus Solier, 1849. Género con unas pocas especies en Nueva Zelanda, tres en Chile y una en Perú.

\section{BROSCINAE \\ BROSCINI}

1- Esclerito glosal con cuatro setas, diente del mentón simple ..... Creobiina .................................................. 2

1' - Esclerito glosal con dos setas, diente del mentón bífido en el ápice .................................................................. 4

2- Tres setas supraorbitales; cinco setas pronotales; nueve a doce setas en la serie umbilical ...... Creobius

2'- Una seta supraorbital; dos a tres setas pronotales; tres a cinco setas en la serie umbilical

3- Ojos casi planos; parte apical del élitro prolongada hacia abajo; machos sin pelos adhesivos en el tarso medio; protarsitos 1-2 ó 1-3 con pelos adhesivos ventralmente ................... Nothocascellius

3'- Ojos convexos; parte apical del élitro no prolongada hacia abajo; macho con setas adhesivas en los mesotarsitos 1-3 y en los protarsitos 1-4 Cascellius

4- Ojos escotados, mentón sin fóveas paramedianas ............................. Barypina ................................................. 5

4'- Ojos no escotados, mentón con dos fóveas paramendianas .............. Nothobroscina ....................... Nothobroscus

5- Submentón con dos setas; mesotarsitos del macho sin pelos adhesivos ventralmente; esternitos abdominales 5-7 con un surco basal completo

\section{Barypus}

5'- Submentón con seis setas; machos con los mesotarsómeros con pelos adhesivos; esternitos abdominales 5-7 sin surco ............................................................................................ Microbarypus

(46) Barypus Dejean 1828. Género distribuido en Chile, Argentina y Uruguay. Veintidos especies agrupadas en tres subgéneros. En Chile están presentes dos subgéneros, uno de ellos con dos especies es endémico de la región central y de la Araucanía (Arathymus Guérin-Ménéville, 1838). El otro subgénero (Cardiopthalmus Curtis, 1839) posee tres especies en Chile, en las regiones de estepa patagónica. Revisiones por Roig-Juñent \& Cicchino (1989) y RoigJuñent (1992). Estudios filogenéticos Roig-Juñent (1995a, 2000).

(47) Creobius Guérin-Ménéville 1838. Género monoespecífico, Creobius eydouxii (Guérin-Ménéville 1838), de la Selva Valdiviana y región de la Araucanía. Revisión y análisis filogenético por Roig-Juñent (1995b, 2000).

(48) Cascellius Curtis 1839. Dos especies, ambas presentes en Chile, distribuidas en bosques de Nothofagus desde la Selva Valdiviana hasta Magallanes. Revisión y análisis filogenético por Roig-Juñent (1995b y 2000).

(49) Microbarypus Roig-Juñent, 2000. Género monoespecífico, Microbarypus sylvatica Roig-Juñent, 2000, endémico de la Selva Valdiviana. Análisis filogenético por Roig-Juñent (2000).

(50) Nothobroscus Roig-Juñent \& Ball, 1995. Género monoespecífico, Nothobroscus chilensis_Roig-Juñent \& Ball 1995, endémico de la Selva Valdiviana. Análisis filogenético Roig-Juñent (2000).

(51) Nothocascellius Roig-Juñent, 1995b. Dos especies, ambas presentes en Chile, distribuidas en bosques de Nothofagus en la región Magallánica. Revisión y análisis filogenético por Roig-Juñent (1995b, 2000). 
ANEXO 1 (continuación)

HARPALINAE

CHLAENIINI.

(52) Chlaenius Bonelli, 1809. Género cosmopolita, que incluye 19 especies neotropicales. En Chile sólo una especie citada (Chlaenius virescens Chaudoir, 1835), sin localidad precisa.

CNEMALOBINI

(53) Cnemalobus Guérin-Ménéville, 1838. Género restringido a Chile, Argentina y Uruguay, con 24 especies. En Chile se encuentra la mayor diversidad de este género, con 17 especies, de las cuales 15 son endémicas. Su distribución es disyunta en Chile, con un área que va desde Coquimbo hasta la región de la Araucanía y otra en la estepa patagónica de Magallanes. Revisión sistemática por Roig-Juñent (1993, 1994a) y Lagos \& Roig-Juñent (1997). Análisis filogenético por Roig-Juñent \& Flores (1995).

\section{CTENODACTYLINI}

(54) Plagiotelum Solier, 1849. Dos especies, una de Tasmania y una de Chile y Argentina. Liebke (1931) cita Plagiotelum irinum Solier, 1849 para Paraguay, pero es un error porque esta especie está estrictamente restringida a los bosques de Nothofagus de la región de la Araucanía.

\section{HARPALINI}

1- Penúltimo artículo del palpo labial plurisetoso 2

1 '- Penúltimo artículo del plapo labial bi o trisetoso ..................................................................................... 3

2- Machos con los tarsos anteriores y medios no modificados o dilatados y con dos hileras longitudinales de setas adhesivas ventrales ............................................................................ Harpalina ............................. Selenophorus

2'- Machos con los tarsos anteriores y medios dilatados y con numerosas hileras longitudinales de setas adhesivas

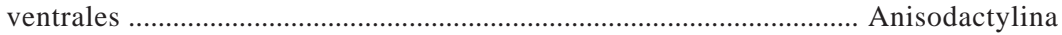

3- Machos con el tarso anterior no modificado o dilatado y con el tarsito basal con dos hileras de setas adhesivas Stenolophina 8

3'- Tarso anterior del macho dilatado, con pubescencia esponjosa abajo Pelmatellina .......................... Trachysarus

4- Pronoto con dos setas laterales en cada lado Allendia

4'- Pronoto con una seta lateral en cada lado 5

5- Mentón y submentón fusionados, no separados por una sutura ......... Anisostichus

5'- Mentón y submentón separados por un surco transverso completo ...

6- Tamaño pequeño, menos de $8,0 \mathrm{~mm}$; metatórax reducido, metepisterno muy corto; alas reducidas; cuerpo despigmentado Nemaglossa

6'- Tamaño mayor, al menos 8,0 mm; metatórax y alas completamente desarrolladas o reducidas; cuerpo de varios colores, muchas especies con al menos el dorso metálico, cobrizo, verde, azul o púrpura ................................ 7

7- Disco del pronoto glabro, excepto la única seta lateral a cada lado; esternitos abdominales con o sin extra setas Notiobia

7'- Disco del pronoto pubescente, al menos a lo largo de la parte lateral del márgen apical; esternitos abdominales con setas extra Criniventer

8- Mentón con diente Acupalpus

8'- Mentón sin diente

9- Basitarso y tarsómero 2 del pretarso asimétricos, ángulo apical interno como un proceso espiniformePolpochila

9'- Basitarso y tarsomero 2 simétricos, ángulo apical interno no extendido ..... 10

10- Tamaño grande, $10 \mathrm{~mm}$ o más; estría escutelar presente; serie umbilical con la seta 6 tan cerca de la 5 como ésta de la 4 ; Paramecus

10' - Tamaño menor, menos de $6 \mathrm{~mm}$; estría escutelar ausente; serie umbilical con la seta 6 alejada de la 5, mucho más que la distancia entre la 5 y 4 Bradycellus

(55) Acupalpus Latreille, 1829. Género de distribución mundial, las 15 especies neotropicales se agrupan en el subgénero Acupalpus (Noonan, 1976). En Chile hay tres especies endémicas.

(56) Allendia Noonan, 1974. Género monoespecífico, Allendia chilensis (Dejean, 1829), de Argentina y Chile. Restringido a la Selva Valdiviana. Descripción y relaciones filogenéticas por Noonan (1974).

(57) Anisostichus van Emdem, 1953. Cuatro especies, dos presentes en Chile desde Chiloé hasta Valparaiso. Revisión por van Emdem (1953).

(58) Bradycellus Erichson, 1837. Posee 16 especies neotropicales. En Chile hay seis especies, una endémica, desde Atacama hasta Puerto Edém.

(59) Criniventer van Emdem, 1953. Género monoespecífico, Criniventer rufus (Brullé, 1838), distribuido en Chile, Argentina y Uruguay.

(60) Nemaglossa Solier, 1849. Género monoespecífico de Chile (Nemaglossa brevis Solier, 1848), de la región de la Araucanía.

(61) Notiobia Perty, 1830. Incluye 25 especies neotropicales. En Chile, cinco especies, tres de ellas endémicas. Revisión por Noonan (1981a). 
ANEXO 1 (continuación)

(62) Paramecus Dejean, 1829. Género de tres especies, una presente en Chile, Paramecus laevigatus Dejean, desde Coquimbo hasta la región de la Araucanía. Sin revisión.

(63) Polpochila Solier, 1849. Género neotropical con 19 especies. En Chile hay dos especies del subgénero Polpochila (Polpochila). Revisión por Nègre (1963).

(64) Selenophorus Dejean, 1829. Este género incluye 149 especies neotropicales, de las cuales sólo hay una presente en Chile (Selenophorus (Selenophorus) variabilis Curtis, 1839).

(65) Trachysarus Reed, 1874. Género casi exclusivo de Chile, con ocho especies en las Islas Juan Fernández y una en la Selva Valdiviana (presente en Argentina, Nègre, 1973), que probablemente no pertenezca a este género (Noonan, 1976). Revisión del género por Straneo \& Jeannel (1955).

\section{LEBIINI}

1- Cabeza ventralmente con al menos un par de puntuaciones setíferas suborbitales

Pericalina .......................

1'- Cabeza ventralmente sin puntuaciones setíferas suborbitales ................................................................... 3

2- Mentón con diente; uñas lisas ........................................................ Catascopellus

2'- Mentón sin diente; uñas pectinadas ................................................ Coptodera

3- Antenómeros 5-11 con pequeñas áreas sensitivas ventrales, caracterizadas por un conjunto denso de setas; coloración del cuerpo y apéndices naranja, élitros con una mácula negra humeral y una banda subapical transversal de color negro ....................................................................... Metallicina ........................... Euproctinus

3' - Antenómeros 5-11 sin áreas sensitivas ventrales; coloración de otra forma ...................................................... 4

4- Mandíbula ensanchada en la base, escroba ancha, márgenes laterales marcadamente redondeados...................... Calleidina ................................................... 6

4'- Mandibula no ensanchada en forma conspicua en la base, escroba estrecha, márgenes laterales no redondeados

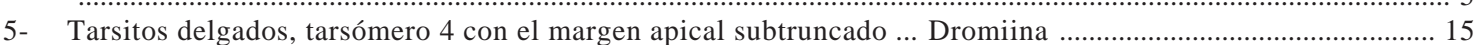

5'- Tarsitos robustos, dilatados, tarsómero 4 bilobado ............................ Lebiina ............................................... Lebia

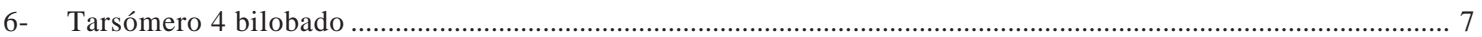

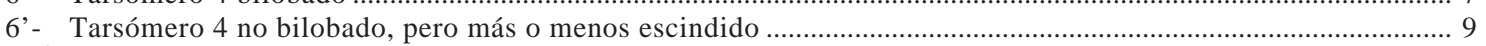

7-Último palpómero maxilar con una excavación cerca del ápice ............. Chaudoirina

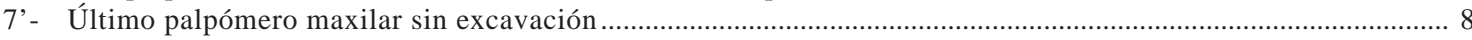

8- Tarsómeros sulcados y glabros dorsalmente ...................................... Calleida

8' - Tarsómeros lisos y pubescentes dorsalmente ..................................... Titaresius

9- Tarsómero 4 profundamente emarginado, pero no bilobado .............. Plochionus

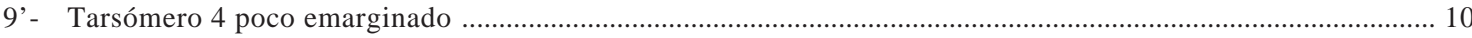

10- Cabeza, pronoto y élitros de coloración azul metálica sin manchas .................................................................... 11

10' - Nunca la coloración como la anterior; a veces el élitro azul metálico, pero entonces la cabeza y el pronoto son negros o pardo-rojizos. La mayoría de las especies de color castaño amarillento con manchas en los élitros .............. 13

11- Antenas y patas completamente negras ............................................ Mimodromites (parte)

11 ' - Apéndices amarillo rojizos o rojo oscuro, a veces el fémur negro, pero el resto de la pata es rojo oscuro ........ 12

12- Alas metatorácicas desarrolladas; largo 5-5,5 mm ............................ Falsodromius

12' - Áptero; largo mayor de $8 \mathrm{~mm}$............................................................. Cyanotarsus

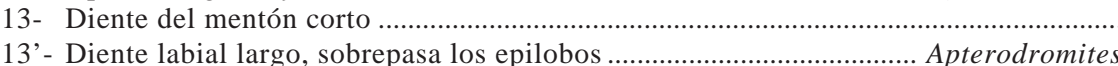

14- Esclerito glosal con cuatro setas, a veces dos apicales bien visibles y dos centrales pequeñas; primer artejo del metatarso más largo que el oniquio ................................................ Mimodromites (parte)

14' - Esclerito glosal con dos setas; primer artejo del metatarso más corto que el oniquio ....................... Mimodromius

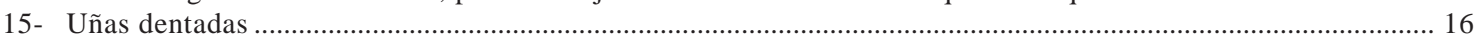

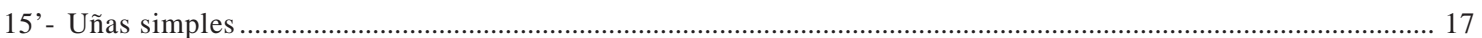

16- Mentón sin diente; serie umbilical formada por seis setas humerales y cuatro apicales; tarsomero 4 alargado y no bilobado Dromius

16'- Mentón con diente; serie umbilical con más de 15 setas; tarsomero 4 aplastado y bilobado Mimophilorhizus

17- Mentón sin diente; último palpomero maxillar oval; tarsos gráciles, los élitros cubren el abdomen; tarsomero 4 escotado Oxoides

17' - Diente del mentón poco desarrollado pero presente; último palpómero maxilar cilíndrico y delgado; élitros cortos que no cubren el abdomen; tarsómero 4 no escotado Axinopalpus

(66) Apterodromites Mateu, 1976. Género monoespecífico, Apterodromites saizi Mateu, 1976, endémico de la región central de Chile.

(67) Axinopalpus LeConte, 1848. Género principalmente neártico con 10 especies neotropicales. Siete especies citadas para Chile, seis de ellas endémicas de Chile Central e Isla Juan Fernández.

(68) Calleida Dejean, 1825. Género cosmopolita con 171 especies neotropicales. En Chile hay dos especies. 
ANEXO 1 (continuación)

(69) Catascopellus Straneo 1969. Género monoespecífico, Catascopellus crassiceps Straneo, 1969, de Chile, de la región de la Araucanía. Revisión por Straneo (1969) y Ball (1975).

(70) Chaudoirina Mateu, 1954. Género con cuatro especies, tres presentes en Chile, desde Coquimbo hasta la región de la Araucanía. Revisión por Mateu (1954).

(71) Coptodera Chaudoir, 1837. Con 40 especies species neotropicales. Dos especies endémicas en Chile.

(72) Cyanotarsus Reed, 1874. Género endémico de Chile Central, con dos especies.

(73) Falsodromius Mateu, 1976. Género monoespecífico, Falsodromius erythropus (Solier, 1849), de Argentina y Chile (Chile central)

(74) Dromius Bonelli, 1809. Género de distribución mundial, con nueve especies en el neotrópico. Para Chile hay citadas cuatro, dos de ellas endémicas. Se distribuye en la región Central y de la Araucanía.

(75) Euproctinus Leng \& Mutchler, 1927. Se extiende desde California hasta Chile, con 16 especies neotropicales. En Chile sólo una especie, Euproctinus (Euproctinus) fasciatus (Solier, 1849), desde Chile Central hasta la región de la Araucanía. Revision y análisis filogenético por Shpeley (1986).

(76) Lebia Latreille, 1802. Género de distribución mundial, con 415 especies en el Neotrópico. En Chile hay tres especies, una aparentemente endémica, Lebia azurea Solier, 1849, de Valdivia y Cautín. Straneo (1969: 872) cita un ejemplar determinado por Mateu como Syntomus platensis Chaudoir, de Chile Chico (Chile). Sin embargo, este género no está presente en América del Sur (Reichardt 1977) y es muy probable que sea una determinación erronea por Lebia platensis Chaudoir, 1871. Si esto es correcto el número de especies de Lebia en Chile sería cuatro.

(77) Mimodromius Chaudoir, 1873. Género de la región austral y andina de América del Sur, con 35 especies. Cinco subgéneros, de los cuales hay presentes en Chile tres, con 15 especies, nueve de ellas endémicas de la región de Atacama. Al Sur, su distribución llega hasta la Selva Valdiviana. Revisiones realizadas por Mateu (1955, 1959).

(78) Mimodromites Mateu, 1955. Género neotropical con cinco especies agrupadas en tres subgéneros. En Chile están presentes las cinco especies, tres de ellas endémicas. Se distribuye desde Coquimbo hasta Magallanes. Revisión por Mateu (1955).

(79) Mimophilorhizus Mateu, 1993. Género monoespecífico de Chile (Mimophilorhizus chilensis Mateu, 1993) distribuido en la región de la Araucanía.

(80) Oxoides Solier, 1849. Monoespecífico, Oxoides obscura Solier, 1849, de Valdivia en Chile.

(81) Plochionus Latreille and Dejean, 1824. Género del hemisferio occidental; en el Neotrópico, 14 especies; en Chile, una sola especie, Plochionus monogrammus Chaudoir, 1877.

(82) Titaresius Liebke, 1935. Género monoespecífico de América meridional. Liebke (1935) no da más referencias de localidad, por lo que no es posible establecer si este género está en Chile, Argentina o en ambos países.

\section{LICININI}

(83) Eutogenius Solier, 1849. Género con dos especies descritas para Chile, Eutogenius fuscus Solier, 1849 y E. magellanicus Philippi, 1862. Solier (1849) no da localidad precisa y dice que habita toda la República, mientras que Philippi (1862) cita su especie del Estrecho de Magallanes.

\section{MASOREINI}

(84) Tetragonoderus Dejean, 1829. Género con 38 especies neotropicales. Dos especies citadas para Chile.

\section{PTEROSTICHINI}

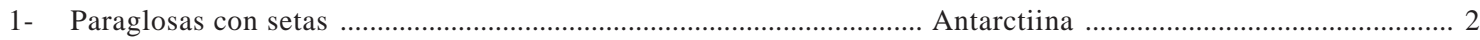

1'- Paraglosas sin setas ..................................................................... Pterostichina ............................................ 4

2- Penúltimo palpómero labial con una hilera de setas (más de dos); cuerpo muy convexo, corto y robusto ............ Kuschelinus

2'- Penúltimo palpómero labial bisetoso; cuerpo alargado, delgado y menos convexo ............................................. 3

3- Tarsómero 4 marcadamente bilobulado ................................................. Abropus

3'- Tarsómero 4 no bilobulado .............................................................. Metius

4- Esternitos IV-VI cada uno con un surco transverso ampliamente interrumpido en la región media (completo en

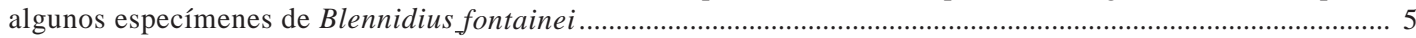

4' - Esternitos IV-VI sin surco transverso y borde, con o sin impresiones longitudinales irregulares en los costados

5- Surcos de los esternitos IV-VI con una hilera de puntos anchos profundos; metepisterno corto o moderadamente alargado Ogmopleura

5'- Surcos de los esternitos IV-VI lisos, sin hilera de puntos profundos, metepisterno alargado, con el borde lateral 1,5 veces más largo que el borde anterior Blennidus

6- Intervalo elitral 3 sin setas ............................................................. Feroniola

6'- Intervalo elitral 3 con al menos una puntuación setífera

7- Proceso intercoxal del prosterno con dos puntuaciones setíferas cerca del ápice.

Parhypates (parte)

7' - Proceso intercoxal glabro, sin puntuaciones setíferas 
8- Metepisterno corto, márgenes anterior y lateral subiguales Parhypates (parte)

8' - Metepisterno alargado, margen lateral más largo que el ancho del borde anterior Trirammatus

(85) Abropus Waterhouse, 1842. Género monoespecífico, Abropus carnifex (Fabricius, 1775), distribuido en los bosques de Nothofagus desde el estrecho de Magallanes hasta Angol. Revisión por Straneo (1951).

(86) Blennidius Motschulsky, 1865. Género de la región austral de América del Sur que llega hasta Bolivia, con 16 especies. En Chile dos especies. Revisión por Straneo (1986).

(87) Feroniola Tschitscherine, 1900. Diez especies; en Chile cuatro, especies distribuidas desde Valparaíso hasta Magallanes. Revisión por Straneo (1995).

(88) Kuschelinus Straneo, 1963. Género monoespecífico, Kuschelinus insularis Straneo, 1963, de las Islas San Ambrosio y San Felipe (800 Km al Norte de Juan Fernández). Revisión por Straneo (1963).

(89) Metius Curtis, 1839. Género de distribución austral en América del Sur, con 62 especies, de las cuales hay 21 en Chile (12 endémicas), distribuidas desde Coquimbo hasta Magallanes. Revisión por Straneo (1951).

(90) Ogmopleura Tschitscherine, 1898. Género distribuido en Perú, Chile, Argentina y Uruguay, con 28 especies. En Chile, seis especies, cinco de ellas endémicas, desde Coquimbo a Concepción.

(91) Parhypates Motschulsky, 1865. Género de distribución austral en América del Sur. En Chile hay 14 especies, ocho de ellas endémicas, distribuidas desde la región de la Araucanía hasta Magallanes.

(92) Trirammatus Chaudoir, 1838. Género de la región austral de América del Sur, con 19 especies. En Chile hay presentes 16 especies, diez endémicas, distribuidas desde Atacama hasta Magallanes. Revisión por Straneo (1969).

\section{PLATYNINI}

1- Meso y metatarsitos sin surcos y dorsalmente con pubescencia....... Pristonychus

1- Meso y metatarsitos con surco (dorsal o lateral) y dorsalmente sin pubecencia .....

2- Uñas tarsales simples

2'- Uñas tarsales pectinadas Incagonum

(93) Calathus Bonelli, 1810. Género neártico, con 13 especies en México. En Chile, Calathus melanocephalus Chaudoir, encontrado en Valparaíso y probablemente introducido.

(94) Pristonychus Dejean, 1828. Una especie en Chile, Pristonychus (Laemosthenes) complanatus Dejean, 1828, de distribución cosmopolita.

(95) Incagonum Liebherr, 1994. Género creado para las especies de América del Sur de Agonum. Incluye 19 especies, 9 de las cuales están en Chile y siete son endémicas, distribuidas desde Chile Central hasta Magallanes. Revisión por Liebherr (1994). 